\title{
UNIVERSITÉ DE SHERBROOKE
}

\section{THÈSE PAR ARTICLES}

PRÉSENTÉE À

\section{LA FACULTÉ DES LETTRES ET DES SCIENCES HUMAINES}

COMME EXIGENCE PARTIELLE

DU DOCTORAT EN PSYCHOLOGIE

PAR

CVÉRONIQUE NEUVILLE

\section{L'IMPACT DES RELATIONS PRÉCOCES SUR}

LE DÉVELOPPEMENT CÉRÉBRAL :

LE TROUBLE RÉACTIONNEL DE L'ATTACHEMENT VU PAR LES NEUROSCIENCES 


\section{Composition du jury}

\section{L'impact des relations précoces d'attachement sur le développement cérébral :}

le trouble réactionnel de l'attachement vu par les neurosciences

Cette thèse a été évaluée par un jury composé des personnes suivantes :

Guadalupe Puentes-Neuman, directrice de recherche (Département de psychologie, Université de Sherbrooke)

Miguel M. Terradas, co-directeur de recherche (Département de psychologie, Université de Sherbrooke)

Claud Bisaillon, membre du jury (Département de psychologie, Université de Sherbrooke)

Jean-François Bureau, membre du jury (Département de psychologie, Université d’Ottawa) 


\section{Sommaire}

L'importance de la qualité des relations précoces parent-enfant pour le développement de l'individu n'est plus à démontrer. Le concept d'attachement se retrouve au cœur de plusieurs théories et de nombreuses recherches concernant l'adaptation affective et sociale de l'enfant, l'adolescent et l'adulte. Qui plus est, ce concept a été emprunté afin de décrire des entités diagnostiques faisant référence à des perturbations dans les relations sociales précoces. Récemment, les critères diagnostiques du trouble réactionnel de l'attachement (TRA) ont subi un remaniement dans la cinquième version du Manuel diagnostique et statistique des troubles mentaux (DSM-5; American Psychiatric Association; APA, 2013) après avoir était relativement stables dans les versions précédentes. En effet, le DSM-5 propose un seul type de TRA, qui correspond au type inhibé de la quatrième version révisée, au lieu de distinguer entre deux types, soit inhibé et désinhibé (DSM-IV-TR; APA, 2000). L'attachement est un domaine prolifique au niveau de la recherche et le développement de l'intérêt des chercheurs concernant le TRA est indéniable. Actuellement, depuis l'émergence des nouvelles technologies d'imagerie médicale, un courant de recherche tend à intégrer ces résultats aux nouvelles connaissances en neuroscience. Ce nouveau champ de recherche tente d'expliquer certains phénomènes par des changements, modifications ou perturbations des structures et connexions cérébrales. De nouvelles perspectives s'ouvraient donc quant à la compréhension de l'impact des troubles d'attachement, et plus particulièrement, du TRA, sur le développement de l'enfant. En effet, actuellement, les troubles d'attachement et le TRA ne représentent pas des entités diagnostiques comparables puisqu'aucune correspondance entre le TRA et les patrons d'attachement traditionnellement décrits en psychologie du développement n'a pu être établie jusqu'à présent (Coleman, 2003; Minnis, Marwick, Arthur, \& McLaughlin, 2006; Newman \& Mares, 2007; 
O’Connor \& Zeanah, 2003). L'étude effectuée par Zeanah, Smyke, Koga et Carlson (2005) suggère que, dans une population d'enfants roumains institutionnalisés, le TRA est associé au degré de formation du lien d'attachement plutôt qu'à l'organisation d'un type particulier d'attachement. Peu de recherches décrivent cependant les représentations d'attachement caractérisant les enfants ayant reçu ce diagnostic. Dans ce contexte, avoir une perspective plus claire des impacts neurobiologiques possibles peut amener une vision plus précise et uniformisée de la compréhension des relations précoces d'attachement perturbées ainsi que des avenues d'intervention. La recherche actuelle suggère la présence de perturbations, pouvant devenir permanentes, des systèmes responsables de régulation des émotions, telles que la peur et l'anxiété, ainsi que des capacités d'attention, du contrôle comportemental, des capacités réflexives et des apprentissages. Dans un premier article, une synthèse des connaissances concernant l'impact des relations d'attachement perturbées sur le développement cérébral et la santé mentale de l'individu est présentée. Pour ce faire, une recension des études reliant les structures cérébrales et les fonctions dont elles sont responsables et décrivant l'impact du TRA au niveau neurobiologique a été effectuée. Cette recension des écrits a permis d'établir des liens potentiels entre le TRA et des altérations au niveau neurodéveloppemental. Dans le second article, l'analyse de trois cas d'enfants d'âge scolaire ayant reçu un diagnostic de TRA est présentée. Cette analyse a le but d'illustrer les prédictions des recherches en neuroscience et d'apporter un éclairage différent à la compréhension de cette pathologie. Les comportements et la symptomatologie des enfants ont été évalués par le biais d'un questionnaire et l'étude des dossiers pédopsychiatriques. La qualité de la relation parent-enfant a été abordée par l'entremise d'un outil permettant d'accéder à leurs représentations d'attachement. L'évaluation des représentations a permis de préciser le profil d'attachement de chaque enfant et de faire émerger plusieurs voies de réflexions concernant la population d'enfants présentant un TRA. En effet, 
l'analyse des résultats laisse entrevoir la possibilité d'un attachement insécurisé chez les enfants étudiés. Elle indique également la présence de difficultés de régulation du comportement et des émotions, des retards d'apprentissage, des problèmes dans les relations interpersonnelles et des déficits attentionnels chez les trois participants. Selon les résultats des études en neuroscience, ces difficultés peuvent être prédites à partir des relations d'attachement perturbées. Ces résultats relient la symptomatologie du TRA à des perturbations dans le développement et le fonctionnement de plusieurs structures cérébrales, dont l'axe HPA (axe hypothalamo-pituitosurrénal) et le cortex frontal. Cette étude permet donc de présenter une vision plus intégrée des éléments affectifs, relationnels et neurologiques qui caractérisent les enfants présentant des relations d'attachement perturbées.

Mots clés : Trouble réactionnel de l'attachement, neurosciences, psychopathologie, enfants 


\section{Table des matières}

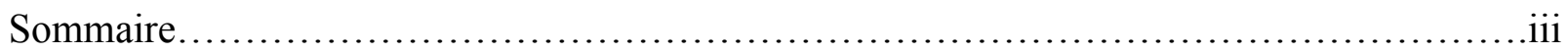

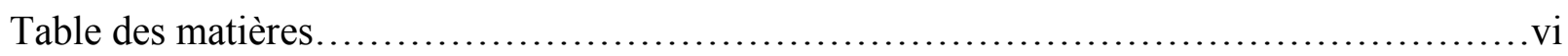

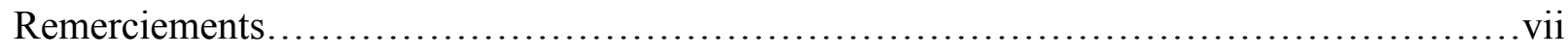

Avant-propos................................................................

Premier Article

L’impact des relations précoces sur le développement cérébral : le trouble réactionnel

de l'attachement vu par les neurosciences...................................... 10

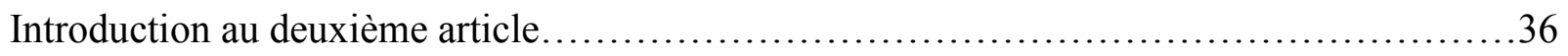

Deuxième article

Convergence entre clinique et neurosciences chez le trouble réactionnel de

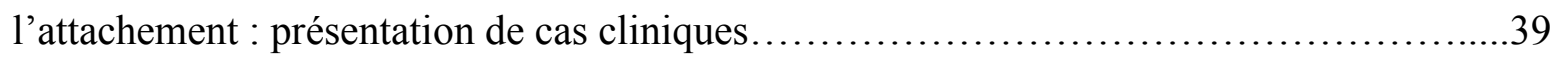

Conclusion................................................................ 74

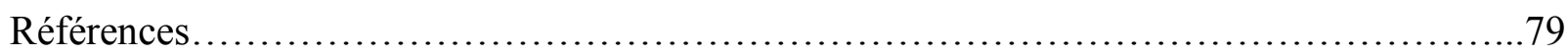

Appendices.................................................................. 85

Appendice A : Exemple d'une histoire des Histoires à compléter........................86

Appendice B : Liste de vérification du comportement des jeunes de 6 à 18 ans

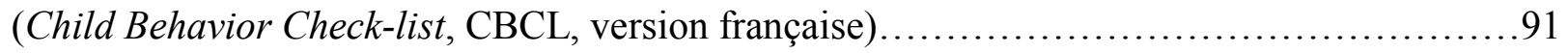




\section{Remerciements}

Ce travail a pu être achevé grâce à la contribution, la patience et la bienveillance de plusieurs personnes. Tout d'abord, je tiens à remercier chaleureusement ma directrice, Madame Guadalupe Puentes-Neuman, et mon co-directeur, Monsieur Miguel M. Terradas, sans qui je ne serais jamais parvenue à venir à bout de ce grand projet. Ils ont fait montre d'une patience infinie, ont su me guider et me conseiller et je leur en suis très reconnaissante. Je remercie également Madame Claud Bisaillon et Monsieur Jean-François Bureau d'avoir accepté de participer à l'évaluation de ce travail; leurs précieux conseils et commentaires m'ont permis d'achever celuici. Sans le soutien de ma famille et particulièrement de mes parents, Jean-Marc et Denise, ma sœur, Marie-Agnès et son conjoint, Éric, ce défi aurait été difficile. Une pensée particulière est adressée à Monsieur Jean Di Persio qui m’a guidée et conseillée durant ce projet ainsi qu'à Nicole pour ses encouragements. Je suis également très reconnaissante envers mon ex-conjoint, Nicolas, et mes enfants, Élisa et Valentin, qui ont dû souvent faire les frais de ma disponibilité limitée et qui se sont montrés très patients lors de mon retour aux études et du processus de rédaction de la thèse. Un grand merci également à Ève-Annick pour son amitié à toute épreuve, son soutien indéfectible, sa patience et son aide, ainsi qu'à Hélène, Christiane, Marianne et Sonia qui ont eu la patience de m'écouter tout au long de ce travail.

Finalement, cela n'aurait pu être possible sans la précieuse collaboration des enfants participants et de leur famille, ni sans la contribution et la collaboration de l'équipe de pédopsychiatrie de l'Hôpital Charles LeMoyne, et tout particulièrement du Dr Claude Grenier, pédopsychiatre. Je vous remercie tous grandement. Merci également à Monsieur Jacques Bigras qui a su me redonner de la motivation au moment où le découragement pointait son nez. 
Avant-propos 
L'attachement est un champ de recherche fécond et stimulant depuis les premiers travaux de Bowlby au milieu du siècle dernier. Il sera d'ailleurs le premier à postuler que l'attachement sélectif précoce est la base indispensable pour le développement de relations sociales ultérieures fonctionnelles puisqu'il permet la régulation de la sécurité et de la survie de l'enfant (Bowlby, 1969, 1973, 1980; Main, 1998). Par la suite, l'opérationnalisation des comportements d'attachement par Ainsworth, Blehar, Waters et Walls (1978), à l'aide du protocole de la Situation étrange a permis d'identifier trois patrons de réponses chez les enfants soumis à ce protocole. Les patrons d'attachement suivants sont décrits : sécurisé (de type B), insécuriséévitant (de type A), insécurisé-résistant (de type C) et, plus tard, le patron désorganisé (de type D) (Main, 1998; Main \& Solomon, 1990). Ainsi, les enfants ayant un patron d'attachement de type sécurisé (de type B) seront des enfants qui, en activant leurs système d'attachement (pleurs, protestations) lors d'une situation alarmante (p. ex., la séparation d'avec la mère) favorisent le rapprochement avec la figure d'attachement et s'apaisent à cette réunion pour ensuite poursuivre leurs jeux ou exploration. En revanche, les enfants décrits comme insécurisés-évitants (de type A) semblent désactiver leur système d'attachement : ils ignorent leurs figures d'attachement lors de la séparation et évitent les tentatives de contact lors de la réunification. Les enfants dont le patron d'attachement est de type insécurisé-résistant (de type C) semblent, quant à eux, hyper-activer leur système d'attachement (p. ex., réagir à des indices mineurs d'insécurité) puisque les réponses maternelles sont généralement inconsistantes. Ils sont alors difficiles à calmer lors des séparations. Ils se montrent donc incapables d'utiliser leur mère comme base sécurisante lors des retrouvailles ce qui les empêche de jouer librement ou d'explorer. Les enfants reconnus comme ayant un patron d'attachement désorganisé (de type D) présentent des comportements désorganisés et/ou désorientés en présence du parent (p. ex., immobilité figée comme en transe, comportements contradictoires d'approche ou de fuite). Ces comportements témoignent d'un 
conflit puisque la figure d'attachement est potentiellement source de confort et de détresse en même temps. La prévalence de ces patrons d'attachement se répartit dans la population générale avec une majorité d'enfants présentant un patron d'attachement sécure, soit entre 62 à $66 \%$. En outre, 15 à $22 \%$ des enfants présenteraient un patron insécure-évitant, 9 à $14 \%$ auraient un patron insécure-ambivalent alors qu'environ $15 \%$ présenteraient un patron désorganisé (Ainsworth et al., 1978; van IJzendoorn \& Kroonenberg, 1988; van IJzendoorn, Schuengel, \& Bakermans-Kranenburg, 1999). Le patron d'attachement désorganisé est d'ailleurs considéré comme un facteur de risque majeur associé au développement d'une psychopathologie chez l'enfant (Borelli, David, Crowley, \& Mayes, 2010; Boris, Fueyo, \& Zeanah, 1997, Green \& Goldwyn, 2002; Lyons-Ruth, 1996; Zeanah, Boris, \& Larrieu, 1997; Zeanah, Boris, \& Scheeringa, 1997). Une entité diagnostique semble conceptuellement liée à la désorganisation de l'attachement, il s'agit du trouble d'attachement. Cependant, bien que très perturbés, les enfants présentant un patron désorganisé ne développeront pas forcément un trouble d'attachement. La désorganisation de l'attachement est donc considérée comme distincte du trouble d'attachement mais constituant plutôt un facteur de risque (van IJzendoorn \& Bakermans-Kranenburg, 2003).

Le trouble d'attachement surviendrait lorsque l'enfant ne peut développer un lien significatif avec une personne disponible et sensible, de par l'absence ou le changement répété de figure de soins en bas âge. Bien que la maltraitance puisse survenir en même temps que des négligences graves ou des changements de soignant, elle serait insuffisante pour expliquer un trouble d'attachement (Hanson \& Spratt, 2000). C'est dans la troisième version du Manuel diagnostique et statistique des troubles mentaux (DSM-III, American Psychiatric Association; APA, 1980) qu'apparaît le diagnostic de trouble réactionnel de l'attachement (TRA) suite à de nombreuses recherches concernant les enfants négligés et vivant en institution (Richters \& 
Volkmar, 1994). Sont alors définis deux types de TRA décrivant un mode de relation sociale gravement perturbé et inapproprié au stade de développement de l'enfant, soit le TRA de type inhibé et désinhibé. Le TRA de type inhibé désigne un enfant présentant une incapacité persistante à s'engager dans des interactions sociales ou y répondre de manière appropriée, se traduisant par des réponses hypervigilantes ou ambivalentes et contradictoires vis-à-vis des adultes lui procurant des soins. Le TRA de type désinhibé décrit des enfants présentant des liens d'attachement diffus, se manifestant par une sociabilité indifférenciée et une incapacité marquée à faire preuve d'attachements sélectifs (Boris, Zeanah, Larrieu, Scheeringa, \& Heller, 1998; Coleman, 2003; Minnis et al., 2006; O’Connor \& Zeanah, 2003; Smyke, Dumitrescu, \& Zeanah, 2002).

Les critères diagnostiques du DSM se sont vus modifiés dans la cinquième version de ce manuel (DSM-5; APA, 2013) et seul demeure le type inhibé des versions précédentes comme l'entité diagnostique associée au TRA. Le type désinhibé constitue maintenant un autre diagnostic à part entière, soit le trouble du contact social désinhibé (TCSD). Le TRA et le TCSD sont donc actuellement considérés comme deux diagnostics distincts et décrits comme des troubles reliés aux traumatismes et aux stresseurs. Le TRA demeure cependant un sujet de controverse et ressemble, pour certains auteurs, aux comportements des enfants ayant formé un patron d'attachement désorganisé (van IJzendoorn \& Bakermans-Kranenburg, 2003), alors que pour d'autres, il constitue un indice d'un attachement non-formé, l'enfant n'ayant pas eu accès à une figure de soin stable (Gleason \& Zeanah, 2010).

La question concernant la correspondance entre le TRA et les patrons d'attachement traditionnellement décrits en psychologie du développement demeure (Coleman, 2003; Minnis et 
al., 2006; Newman \& Mares, 2007; O’Connor \& Zeanah, 2003) et l'étude effectuée par Zeanah, Smyke, Koga et Carlson (2005) suggère que, dans une population d'enfants roumains institutionnalisés, le TRA est associé au degré de formation du lien d'attachement plutôt qu'à l'organisation d'un type particulier d'attachement. Chez les enfants d'âge scolaire, les comportements d'attachement sont moins perceptibles que chez les jeunes enfants et la procédure de la Situation étrange n'a pas été validée pour les évaluer avec précision. Les représentations d'attachement sont alors utilisées pour décrire les patrons d'attachement des enfants plus âgés. En effet, les expériences précoces avec les figures d'attachement permettent l'émergence progressive d'un système de pensées, de croyances, de souvenirs, d'attentes, d'émotions et de comportements concernant l'individu lui-même et les autres. Ces schèmes relationnels, les modèles opérants internes (MOI), régulent, interprètent et prédisent les comportements lors des relations avec autrui, et en particulier, avec les figures d'attachement (Bowlby, 1973, 1980; Bureau, Béliveau, Moss, \& Lépine, 2006; Main, Kaplan, \& Cassidy, 1985; Splaun, 2012). Une continuité et une corrélation sont effectivement observées entre les patrons d'attachement évalués durant la petite enfance et les représentations d'attachement inscrites dans les MOI étudiés à l'âge scolaire (Bureau \& Moss, 2010). Selon DeKlyen et Greenberg (2008), un attachement insécure aurait un impact important sur la future mésadaptation potentielle de l'individu de par la cristallisation des MOI, modèles caractérisés par de la colère, de la méfiance, de l'anxiété et de la peur. En effet, ces représentations d'attachement influencent l'ensemble des relations de la personne ainsi que ses capacités à négocier avec les diverses événements de sa vie (Bureau et al., 2006; Main et al., 1985).

Les représentations d'attachement des enfants d'âge scolaire sont évaluées grâce aux narratifs d'attachement telles que les Histoires à compléter (Attachment Story Completion Task, 
ASCT; Bretherton, Ridgeway, \& Cassidy, 1990). Lors de cette procédure, l'enfant est invité, à l'aide de figurines, à compléter des histoires dont la mise en situation réfère à des thèmes anxiogènes en lien avec la relation d'attachement aux parents. C'est grâce à cette mise en situation que le système d'attachement est activé. Les histoires élaborées par l'enfant sont réputées être guidées par ses représentations des relations d'attachement avec ses parents. Ainsi, face à une situation nécessitant le soutien ou la protection de l'adulte, l'enfant produira une histoire qui rend compte de ce qu'il peut lui-même attendre dans le contexte de sa relation avec la figure d'attachement. En effet, les représentations d'attachement captées lors des histoires présentées à l'enfant sont conformes avec la relation d'attachement que l'enfant entretient avec ses parents en dehors des récits (Grych, Wachsmuth-Schlaefer, \& Klockow, 2002; Oppenheim, 2006; Steele et al., 2003).

Depuis le début du XXIe siècle, avec l'émergence des neurosciences, une nouvelle perspective concernant l'attachement émerge. En effet, l'établissement de liens d'attachement privilégiés et sécurisés est reconnu comme la base nécessaire pour favoriser le développement optimal de l'enfant autant au niveau physiologique que psychologique et neurologique. Des relations d'attachement sécurisantes participent au bon développement des structures cérébrales (Ludy-Dobson \& Perry, 2010). Il est donc supposé que dans le cas de relations d'attachement altérées, de patrons d'attachement insécurisants ou de troubles d'attachement, des perturbations dans le développement de certaines structures cérébrales pourraient être observées.

En effet, plusieurs études révèlent des altérations du fonctionnement des axes et structures cérébrales participant à la régulation des émotions (l'axe HPA, constitué de l'hypothalamus, l'hypophyse ou glande pituitaire et l'amygdale) chez des enfants vivant des relations 
d'attachement perturbées (Fenoglio, Chen, \& Baram, 2006; Kemeny, 2003; Thomas et al., 2001). De même, certaines études indiquent des perturbations au niveau des capacités d'apprentissage, des processus de mémorisation, du contrôle attentionnel et des habiletés visuo-spatiales chez les individus ayant bénéficié de soins maternels inadéquats (Fenoglio et al., 2006). On signale également des perturbations sur le plan des structures cérébrales supérieurs telles que l'hypothalamus et le cortex frontal, structures participant à la régulation du comportement, des émotions, de l'attention et à l'expression des habiletés sociales (Bretherton \& Munholland, 2008; Chugani et al., 2001).

\section{Contribution de l'auteure}

Les deux articles composant la présente thèse traitent de l'influence des relations d'attachement perturbées sur le développement cérébral de l'enfant présentant un diagnostic de TRA. Le premier article tente de dégager l'impact d'un TRA sur les relations interpersonnelles, les comportements et la régulation des émotions chez l'enfant, tout en considérant ce que la recherche scientifique postule sur les conséquences de ce trouble sur le développement des structures cérébrales. Cet article propose donc d'envisager le TRA selon une perspective neurodéveloppementale, perspective qui considère le cerveau comme étant la structure qui organise le fonctionnement émotionnel, social, cognitif et comportemental de l'individu (Perry, 2008). En effet, des résultats concernant des altérations dans le développement et le fonctionnement de certaines structures cérébrales chez des enfants, adolescents et adultes présentant des perturbations au niveau de l'attachement commencent à émerger et peuvent expliquer certains troubles observés au niveau du contrôle des émotions, du comportement, des apprentissages et des relations avec autrui. Certaines altérations peuvent également se chroniciser et devenir permanente par la suite. Cet article propose un rapprochement entre les résultats des 
recherches en neuroscience et la symptomatologie observée chez les enfants ayant reçu un diagnostic de TRA dans l'optique de décrire les altérations cérébrales possibles que ces enfants pourraient présenter. L'article a été accepté pour publication sans correction majeure par la revue française La psychiatrie de l'enfant. Il sera publié dans le premier numéro de l'année 2018. Voici la référence de cet article :

Neuville, V., Puentes-Neuman, G., \& Terradas, M. M. (sous presse). L’impact des relations précoces sur le développement cérébral : le trouble réactionnel de l'attachement vu par les neurosciences. La psychiatrie de l'enfant.

Le second article propose d'étudier le profil clinique d'enfants ayant reçu un diagnostic de TRA afin de vérifier les similitudes entre les manifestations cliniques et les perturbations neurodéveloppementales possibles pouvant y être associées, tel que prédit par la recherche en neurosciences et décrit dans le manuscrit précédent. Pour ce faire, l'analyse de trois cas d'enfants présentant un diagnostic de TRA est présentée. En plus de l'étude du dossier clinique des enfants, la Liste de vérification du comportement des jeunes de 6 à 18 ans (traduction française du Child Behaviour Checklist, CBCL; Achenbach \& Rescorla, 2001) et le Système de cotation centré sur l'attachement pour les histoires à compléter (Attachment Focused Coding System, AFCS; Splaun, 2012) permettront respectivement de préciser la symptomatologie et les représentations d'attachement des enfants participant à l'étude. Une discussion des cas sera par la suite effectuée à la lumière des connaissances issues de la recherche en neuroscience afin d'expliquer les atteintes neurodéveloppementales pouvant être présentes chez ces enfants. L'article a récemment été soumis à la revue Revue québéquoise de psychologie. Nous sommes en attente des résultats du processus d'évaluation par les pairs. Voici la référence de l'article : 
Neuville, V., Terradas, M. M., Puentes-Neuman, G., \& Grenier C. (soumis). Convergence entre clinique et neurosciences chez le trouble réactionnel de l'attachement : présentation de cas cliniques. Revue québécoise de psychologie. 


\section{Premier article}

(les normes de présentation sont celles de la revue La psychiatrie de l'enfant) 
L'impact des relations précoces sur le développement cérébral : le trouble réactionnel de l'attachement vu par les neurosciences

Véronique Neuville, Guadalupe Puentes-Neuman et Miguel M. Terradas

Note des auteurs

Véronique Neuville, MPs., psychologue, Longueuil, Québec, Canada. Guadalupe PuentesNeuman, PhD., Professeure, Département de Psychologie, Université de Sherbrooke-Campus de Longueuil, Québec, Canada. Miguel M. Terradas, PhD., Professeur et psychologue, Département de Psychologie, Université de Sherbrooke-Campus de Longueuil, Québec, Canada 


\title{
Résumé
}

L'attachement demeure un vaste champ de recherche de par l'importance de la qualité de relations précoces sur le développement de l'individu. Depuis l'émergence des connaissances concernant les neurosciences, de nouvelles perspectives s'ouvrent quant à l'impact des troubles d'attachement, et plus particulièrement, du trouble réactionnel de l'attachement, sur le développement des structures cérébrales de l'enfant. Actuellement, les recherches semblent converger vers des perturbations, pouvant devenir permanentes, des systèmes de régulation des émotions au niveau limbique telles que la peur et l'anxiété, mais également du fonctionnement des structures corticales supérieures altérant les capacités d'attention, le contrôle des comportements, les habiletés sociales, les capacités réflexives et les apprentissages. Cet article présente une synthèse des connaissances actuelles concernant l'impact des relations d'attachement perturbées sur le développement cérébral et la santé mentale de l'individu.

Mots-clés : Trouble réactionnel de l'attachement, neurosciences, psychopathologie.

\begin{abstract}
Attachment and its influence on relational problems remains a vast field of inquiry in developmental research. The emergent area of the neurosciences opens new perspectives into the understanding of the impact of attachment disorders in general, and more specifically, of Reactive Attachment Disorder, on the young child's brain structures. Current research suggests that early socioemotional experiences might impact limbic structures responsible for the regulation of emotions such as fear and anxiety, as well as superior cortical structures responsible for attention, behaviour control, social skills, reflexive capacities, and learning. This article presents current knowledge on the impact attachment relations may have on the development of brain structures and individual's mental health.
\end{abstract}

Key words: Reactive attachment disorder, neurosciences, psychopathology. 
L'impact des relations précoces sur le développement cérébral: le trouble réactionnel de l'attachement vu par les neurosciences

Depuis l'émergence du champ d'étude s'intéressant aux neurosciences, de nombreux chercheurs tentent de déterminer l'impact des relations précoces perturbées sur le développement cérébral et leurs conséquences en ce qui concerne le développement psychologique de la personne (p. ex., Hunter, Minnis, \& Wilson, 2011; Perry, 2001, 2008; Pollan \& Hoffer, 2008). À la lumière des connaissances actuelles, cet article tentera de dégager l'impact d'un trouble réactionnel de l'attachement (TRA) sur les relations interpersonnelles, les comportements et la régulation des émotions chez l'enfant tout en considérant ce que la recherche indique des conséquences de ce trouble sur le développement des structures cérébrales. Bien que de nombreuses descriptions soient disponibles concernant les effets au niveau comportemental, relationnel ou émotionnel de relations d'attachement perturbées, les connaissances concernant l'impact d'un TRA sur le développement du cerveau commencent seulement à émerger. Les quatre premières années de vie sont reconnues comme une période primordiale concernant le développement du cerveau, période lors de laquelle plus de $80 \%$ des changements structuraux majeurs se produisent durant l'organisation cérébrale (Ludy-Dobson \& Perry, 2010). Cette organisation cérébrale, qui se poursuivra jusqu'au début de l'âge adulte, est en partie guidée par la nature des expériences vécues par l'enfant. Ainsi, les expériences dans l'univers socioaffectif, tout comme celles vécues dans l'environnement physique, contribuent à façonner l'organisation cérébrale (Perry, 2001, 2008). Cet article propose d'envisager le TRA selon une perspective neurodéveloppementale, perspective qui considère le cerveau comme étant la structure qui organise le fonctionnement émotionnel, social, cognitif et comportemental de l'individu. En fonction de la nature spécifique des difficultés observées (p. ex., anxiété, inattention, régulation des affects), des perturbations de certains circuits neuronaux et aires cérébrales peuvent être inférées (Perry, 2008).

Dans un premier temps, un bref survol des notions d'attachement, de patrons d'attachement et de troubles d'attachement sera présenté afin de mettre en perspective certaines nuances. Puis, suivra un essai d'intégration des connaissances concernant l'impact du TRA sur les structures cérébrales et le traitement de l'information du cerveau en développement. Les répercussions sur le fonctionnement psychologique de la personne et, plus spécifiquement, sur les 
plans comportemental, émotionnel et de la santé mentale de l'individu y seront également examinées.

\section{QUELQUES NOTIONS CONCERNANT L'ATTACHEMENT}

Pour être en mesure de déterminer les conséquences du TRA sur le développement de l'enfant, un bref survol théorique nous parait nécessaire. John Bowlby (1969) sera le premier à postuler que l'attachement sélectif précoce est la base indispensable pour le développement de relations sociales ultérieures fonctionnelles puisqu'il permet la régulation de la sécurité et de la survie de l'enfant (Main, 1998). Bowlby voyait dans l'étude des relations précoces des enfants avec leurs pourvoyeurs de soins, une des sources les plus importantes d'information sur le développement de leur personnalité future. Par la suite, l'intérêt des scientifiques concernant les relations d'attachement n'a cessé de grandir. C'est ainsi qu'émerge la Situation étrangère, une méthode d'évaluation des patrons d'attachement chez les jeunes enfants, proposée par Ainsworth, Blehar, Waters et Walls (1978), dans le but d'opérationnaliser les comportements d'attachement. Trois patrons de réponses sont alors identifiés chez les enfants soumis à cette procédure. Chacun de ces patrons comportementaux serait un reflet du modèle de représentation interne de l'enfant au sujet de sa relation avec le parent puisque ces représentations internes sont à l'origine des stratégies adaptatives de l'enfant face aux comportements de son parent et des croyances qu'il construit sur lui-même, son donneur de soin et leur relation (Bowlby, 1969, 1973, 1980; Bretherton et Munholland, 2008). Ce modèle représentationnel organiserait l'expérience et la réponse de l'enfant face aux situations sollicitant le système d'attachement. Chez la majorité des enfants étudiés, le patron de réponses sécurisées est observé. Ces enfants utilisent leur mère comme base de sécurité pour l'exploration, tout en manifestant une détresse lors de la séparation et un accueil actif lors de la réunion avec celle-ci (patron sécure, de type B). D'autres enfants, cependant, démontraient des comportements indiquant qu'ils semblaient "préoccupés » par la mère durant toute la procédure en étant soit en colère, passifs ou difficilement consolables après la réunion (patron insécure-résistant, de type $\mathrm{C}$ ). Enfin, plusieurs enfants ne présentaient que peu de réaction de pleurs face à la séparation d'avec la mère et évitaient activement ou ignoraient celle-ci lors de la réunion (patron insécure-évitant, de type A) (Main, 1998). De façon générale, les enfants dont l'attachement est évitant auraient tendance à minimiser l'expression des émotions négatives et les comportements d'attachement en présence du parent puisque, 
d'expérience, ceux-ci ont été majoritairement minimisés ou rejetés par la figure de soins. Les enfants ayant un attachement résistant auraient tendance à amplifier l'expression des émotions négatives et des comportements d'attachement pour tenter d'obtenir l'attention du parent qui, d'ordinaire, répond de façon inconstante. En revanche, les enfants ayant un attachement de type sécure expriment leur détresse à leurs parents qui sont généralement en mesure de leur apporter du réconfort et leur servir de base de sécurité pour l'exploration une fois l'apaisement regagné.

Ces trois patrons différents d'attachement (A, B et C) sont considérés comme des stratégies organisées et adaptatives en fonction de l'environnement dans lequel évolue l'enfant; chacun est supposé favoriser un maximum de proximité avec la figure d'attachement. Cependant, certains enfants ne parvenaient pas à déployer une stratégie d'attachement organisée et ne pouvaient donc être classés dans l'une de ces trois catégories. Ces enfants souvent négligés et maltraités démontraient, lors de la procédure de la Situation étrangère, des comportements anormaux, contradictoires et peu organisés en présence du parent (Ainsworth et al., 1978; Crittenden, 1985; Main \& Solomon, 1990). Une quatrième catégorie est alors définie : le patron insécurisé/désorganisé-désorienté (type D; Main \& Solomon, 1990). Le patron d'attachement désorganisé est décrit par les chercheurs comme un effondrement, parfois momentané, d'une stratégie cohérente et organisée de régulation des émotions afin de composer avec une situation d'attachement stressante comme la séparation ou la réunion d'avec le parent (van IJzendoorn \& Bakermans-Kranenburg, 2003; van IJzendoorn, Schuengel, \& Bakermans-Kranenberg, 1999). Il est proposé que l'enfant présentant un patron d'attachement désorganisé se retrouve dans une situation paradoxale et souvent insoluble, le parent étant à la fois une base de sécurité et source de menace et de violence potentielle (Main \& Hesse, 1990). L'incompatibilité entre la fuite et la recherche de proximité expliquerait l'effondrement caractéristique de l'attachement désorganisé (van IJzendoorn et al., 1999).

\section{DISTRIBUTION ET PRÉVALENCE DES PATRONS D’ATTACHEMENT}

Selon les études (Ainsworth et al., 1978; van IJzendoorn et Kroonenberg, 1988; van IJzendoorn et al., 1999), la prévalence du patron d'attachement sécure dans la population générale se situerait entre 62 à $66 \%$; celle du patron insécure-évitant entre 15 et $22 \%$, et de 9 à $14 \%$ concernant le patron insécure-ambivalent. Pour le patron désorganisé, elle se situerait autour de $15 \%$ de la population «normale », soit une population nord-américaine de classe moyenne ne faisant pas l'objet d'un diagnostic clinique. Le patron d'attachement désorganisé 
demeure cependant surreprésenté chez les enfants abusés et maltraités. En effet, sa prévalence augmente à $43 \%$ chez les familles dans lesquelles les mères présentent des problèmes de dépendance à l'alcool et aux drogues et atteint jusqu'à 48 à $77 \%$ dans les familles de parents maltraitants (Ainsworth et al., 1978; van IJzendoorn et al., 1999).

\section{DÉSORGANISATION DE L’ATTACHEMENT}

Le patron d'attachement désorganisé est considéré comme un facteur de risque majeur associé au développement d'une psychopathologie chez l'enfant (Borelli, David, Crowley, \& Mayes, 2010; Boris, Fueyo, \& Zeanah, 1997, Green \& Goldwyn, 2002; Lyons-Ruth, 1996; Zeanah, Boris, \& Larrieu, 1997; Zeanah, Boris, \& Sheeringa, 1997). Lorsque rencontré à un âge précoce chez l'enfant, il prédirait les problématiques de comportements externalisés et l'agressivité chez ces mêmes enfants à l'âge scolaire (DeKlyen \& Greenberg, 2008; Fearon, Bakermans-Kranenburg, van IJzendoorn, Lapsley, \& Roisman, 2010; Lyons-Ruth, 1996; LyonsRuth, Alpern, \& Repacholi, 1993). De plus, la désorganisation de l'attachement serait un patron d'attachement stable entre l'âge de 1 et 5 ans quelle que soit la classe socio-économique considérée (van IJzendoorn et al., 1999). Le patron d'attachement désorganisé présente également une corrélation positive avec des tendances dissociatives à l'âge de 17 ans (Carlson, 1998). Sroufe, Egeland, Carlson et Collins (2005) signalent que, en ce qui concerne la psychopathologie rencontrée à l'adolescence, le prédicteur le plus puissant des six premières années de la vie est un attachement désorganisé. Selon van IJzendoorn et Bakermans-Kranenburg (2003), la désorganisation de l'attachement pourrait même être considérée comme un signe précoce de psychopathologie. En effet, les perturbations dans les relations d'attachement entraînent des altérations dans les fondements de certains acquis développementaux comme la régulation émotionnelle, pouvant alors mener vers une psychopathologie ultérieure (Egeland \& Carlson, 2004). Notons que, bien que très perturbés, les enfants présentant un patron désorganisé ne développeront pas forcément un trouble de l'attachement. La désorganisation de l'attachement est donc considérée comme distincte du trouble d'attachement, constituant ainsi un facteur de risque (van IJzendoorn \& Bakermans-Kranenburg, 2003).

La «désorganisation» comportementale de l'enfant observée dans le patron d'attachement désorganisé proviendrait d'un modèle opérant interne (MOI) fragmenté où se mêlent des représentations d'un parent étant à la fois source de peur et de réconfort, représentations ne pouvant être intégrées (Main \& Hesse, 1990). En effet, les expériences 
précoces avec les figures de soin permettent l'émergence progressive d'un système de pensées, de croyances, de souvenirs, d'attentes, d'émotions et de comportements concernant l'individu luimême et les autres. Ces schèmes relationnels, les MOI, régulent, interprètent et prédisent les comportements lors des relations avec autrui, et en particulier, avec les figures d'attachement (Bowlby, 1973, 1980; Bureau, Béliveau, Moss, \& Lépine, 2006; Main, Kaplan, \& Cassidy, 1985). Une continuité et une corrélation est effectivement observée entre les patrons d'attachement évalués durant la petite enfance et les représentations d'attachement inscrites dans les MOI étudiés à l'âge scolaire pour une population non-clinique (Bureau \& Moss, 2010). Selon DeKlyen et Greenberg (2008), un attachement insécure aurait un impact important sur la future mésadaptation de l'individu de par la cristallisation des MOI, modèles caractérisés par de la colère, de la méfiance, de l'anxiété et de la peur.

\section{LE TRA : DÉFINITION ET HISTORIQUE}

Le trouble d'attachement peut survenir lorsque l'enfant ne peut développer un lien significatif avec une personne disponible et sensible, de par l'absence ou le changement répété de figure de soins en bas âge. Bien que la maltraitance puisse survenir en même temps que des négligences graves ou des changements de soignant, elle serait insuffisante pour expliquer un trouble d'attachement (Hanson \& Spratt, 2000). La maltraitance serait alors associée à un attachement développé bien que désorganisé. Le trouble d'attachement survient donc dans trois contextes de soins, soit lors de la fréquentation de certaines institutions comme les orphelinats, lors de changements répétés de la figure de soins principale (p. ex., plusieurs familles d'accueil) ou lorsque l'enfant habite avec des soignants identifiables, mais extrêmement négligents.

Depuis la moitié du XX siècle, des cliniciens décrivent des symptômes cliniques chez des enfants exposés à des privations sociales importantes telles que la séparation d'avec la mère chez le nourrisson. Ces enfants présentent en effet d'importantes perturbations au niveau des relations sociales, de la gestion des affects, du développement physique et des réponses immunitaires (Spitz, 1946). La première recherche longitudinale consacrée à l'étude de ces enfants, effectuée par Tizard et Rees en 1975, décrit des perturbations importantes du comportement chez de très jeunes enfants vivant en institution, subissant les effets de privations sociales malgré les soins médicaux et les besoins primaires comblés. Deux troubles de comportement de nature relationnelle ont été ainsi identifiés : le type indiscriminé et le type retiré. Ils seront par la suite utilisés pour décrire le TRA de la troisième version du Diagnostic and Statistical Manual of 
Mental Disorder (DSM-III; American Psychiatry Association; APA, 1980; Gleason et al., 2011) qui fut dès lors reconnu comme un désordre à part entière. Les comportements ici décrits constituent des comportements sociaux en présence de donneurs de soin non-spécifiques et non de la figure d'attachement éventuelle de l'enfant.

Dans sa version initiale, le diagnostic de TRA repose sur l'existence d'un retard de croissance et d'un manque de réponses sociales observables avant l'âge de 8 mois. Lors de la révision de 1987, l'âge d'apparition des symptômes est alors repoussé à 5 ans et deux types sont différenciés, soit inhibé et désinhibé. Par la suite, le DSM-IV (APA, 1994) et le DSM-IV-TR (APA, 2000) associent le TRA à des soins gravement déficients et pathogènes durant l'enfance. Le symptôme prépondérant demeure le mode de relation social gravement perturbé et inapproprié au stade de développement apparu avant l'âge de 5 ans. Le TRA de type inhibé désigne un enfant présentant une incapacité persistante à s'engager dans des interactions sociales avec des adultes lui procurant des soins ou à y répondre d'une manière appropriée au stade du développement. Il se traduit par des réponses excessivement inhibées, hypervigilantes ou nettement ambivalentes et contradictoires. L'enfant ayant un TRA de type désinhibé présente des liens d'attachement diffus, qui se manifestent par une sociabilité indifférenciée et une incapacité marquée à faire preuve d'attachements sélectifs. Des comportements d'attachement tels que la recherche de proximité et de contact avec des personnes non-familières sont alors observés dans des situations ne les sollicitant pas normalement, comportements qui ne parviennent pas à sécuriser l'enfant (Boris, Zeanah, Larrieu, Scheeringa, \& Heller, 1998; Coleman, 2003; Minnis, Marwick, Arthur, \& McLaughlin, 2006; O’Connor \& Zeanah, 2003; Smyke, Dumitrescu, \& Zeanah, 2002).

\section{VALIDITÉ DES CRITÈRES DIAGNOSTIQUES DU TRA}

De nombreuses études portent sur la validité des critères diagnostiques du TRA. En effet, depuis quelques années, les dernières recherches remettent en question la présence d'un trouble composé de deux sous-types en faveur de deux troubles distincts (APA, 2013; Gleason et al., 2011; Gleason \& Zeanah, 2010; Rutter, Kreppner, \& Sonuga-Brake, 2009). Cette distinction se retrouve actuellement dans le DSM-5 (APA, 2013) avec la description de deux désordres reliés aux traumatismes et aux stresseurs, soit le TRA (type inhibé du DSM-IV-TR) et le trouble du contact social désinhibé (TCSD, type désinhibé du DSM-IV-TR). Ainsi, le TCSD n'est plus considéré comme un trouble d'attachement mais comme une perturbation majeure du développement social de l'enfant. Pour certains auteurs, le TRA ressemble davantage aux 
comportements des enfants ayant formé un attachement désorganisé et pourrait être considéré comme une indication de désorganisation extrême de par l'intensité des comportements observés (van IJzendoorn \& Bakermans-Kranenburg, 2003). Pour d'autres auteurs, le TRA constitue un indice d'un attachement non-formé, l'enfant n'ayant pas eu accès à une figure de soins stable (Gleason \& Zeanah, 2010). En revanche, certains symptômes du TRA demeurent non-spécifiques (p. ex., répertoire de réponses sociales et émotionnelles limité, peu d'affects positifs, épisodes d'irritabilité, de tristesse ou de peur) et peuvent également être présents dans d'autres catégories diagnostiques (p. ex., troubles du spectre de l'autisme, trouble de comportement, trouble anxieux, trouble déficitaire de l'attention, trouble de stress post-traumatique) n'impliquant pas nécessairement des relations d'attachement perturbées (Hanson \& Spratt, 2000). Certains enfants peuvent donc recevoir un diagnostic utilisé plus fréquemment, tel que le trouble d'attention ou le trouble de comportement, certains cliniciens demeurant moins familiers avec celui de TRA (Damen, Pütz, Herpetz-Dahlmann, \& Konrad, 2012).

La stabilité dans le temps de cette entité diagnostique a également été mise en question. En effet, s'il s'agit à la base d'un attachement non-formé faute de pourvoyeur de soins identifiable, se pourrait-il que certains enfants accueillis dans un milieu favorable et stable par la suite puissent développer une relation d'attachement et ne plus satisfaire les critères diagnostiques du TRA? (Hanson \& Spratt, 2000). Ce diagnostic peut-il donc être provisoire? Étant donné le manque de données précises concernant l'épidémiologie et l'étiologie du TRA, il s'avère difficile de pouvoir établir un pronostic concernant le devenir de ces enfants ainsi que de déterminer les traitements efficaces (Chaffin et al., 2006). Malgré les modifications apportées aux critères diagnostiques dans la foulée du DSM-5 (APA, 2013), cette classification nosographique demeure une description symptomatologique se basant sur l'observation de comportements sociaux aberrants présentés par les individus et non sur la relation que ceux-ci entretiennent avec une figure particulière, ce qui est au centre de l'attachement (Damen et al., 2012).

Concernant l'épidémiologie, celle-ci demeure également peu connue. Bien que certaines études rapportent qu'environ $20 \%$ des enfants institutionnalisés présentent un TRA de type désinhibé, seulement 3 à $5 \%$ souffriraient de la forme inhibée (Gleason et al., 2011). Avec la nouvelle classification du DSM-5, on pourrait donc s'attendre à ce que 3 à $5 \%$ de la population des enfants placés en institution présente un tel diagnostic. En ce qui concerne l'étiologie, la classification du DSM-5 présuppose que l'enfant ait été soumis à un environnement très 
négligeant ou maltraitant. Cependant, les informations concernant cette période précoce sont généralement transmises par les parents ou les membres des institutions, personnes directement impliquées auprès de l'enfant, ce qui diminue la validité de telles informations. En outre, il est reconnu qu'une relation stable peut être tout aussi toxique et mener à un trouble chez l'enfant. En ce sens, la Classification diagnostique de la santé mentale et des troubles du développement de la petite enfance et l'enfance (Diagnostic Classification of Mental Health and Developmental Disorders of Infancy and Early Childhood; Zero to Three, 1994, 2005) inclut, sur son deuxième axe, la qualité de la relation parent-enfant. Cette dimension est évaluée par les observations du clinicien, les informations obtenues concernant l'expérience subjective des parents et de l'enfant ainsi que les résultats de la complétion d'une échelle, le «Parent-Infant Relationship Global Assessment Scale » (PIR-GAS). Elle permet, en évaluant l'intensité, la fréquence et la durée des difficultés dans une relation, de déterminer s'il est question d'une perturbation, d'un dérangement ou d'un trouble (Aoki, Zeanah, Heller, \& Bakshi, 2002).

D'autre part, le diagnostic de TRA suppose que les critères doivent être observés avant l'âge de 5 ans. Par contre, aucune étude ne détermine l'âge de 5 ans comme un seuil valide pour l'apparition des symptômes (Hanson \& Spratt, 2000). Il demeure donc que le diagnostic de TRA nécessite encore des ajustements ainsi que l'élaboration de grilles plus spécifiques et plus sensibles basées sur l'observation du comportement de l'enfant afin de raffiner l'établissement de ce diagnostic (Zeanah, Boris, \& Lieberman, 2000).

\section{LES CONSÉQUENCES NEUROBIOLOGIQUES DU TRA SUR LE DÉVELOPPEMENT}

\section{CÉRÉBRAL}

L'humain est une créature sociale dont la survie dépend depuis des millénaires de son entourage. L'établissement de liens d'attachement privilégiés et sécuritaires est nécessaire pour favoriser un environnement optimal au développement de l'individu. C'est dans cet environnement chaleureux et sécurisant que l'enfant développera ses capacités de régulation affective et comportementale afin de favoriser le maintien d'un état apaisé propice aux apprentissages et au bon développement cérébral (Ludy-Dobson \& Perry, 2010). En effet, dès les premiers jours de vie, le système cérébral du nouveau-né s'active de façon innée pour favoriser l'installation d'un attachement à la figure principale de soins et diriger son attention vers celle-ci (Polan \& Hoffer, 2008). Dans un contexte relationnel adverse caractérisé par des changements répétés de figure de soins ou de négligence et d'abus, le développement de l'enfant, autant 
physique et émotionnel que neurologique est compromis, l'environnement ne lui permettant pas d'intégrer ni d'autoréguler les expériences de façon adéquate.

\section{L'axe HPA}

L'axe HPA est l'axe essentiel à la régulation du stress chez l'individu. Il implique trois structures cérébrales: 1'hypothalamus, l'hypophyse (glande pituitaire) et l'amygdale. Les relations précoces d'attachement auraient un impact important sur le fonctionnement de l'axe HPA (Fenoglio, Chen, \& Baram, 2006). En effet, en fonction de la qualité et de la quantité des premières interactions avec la figure de soins, la stimulation de l'axe HPA permet de réguler la sensibilité du nourrisson aux événements agressants en intervenant comme un thermostat sur les autres structures cérébrales. C'est en effet grâce à l'hypothalamus que le cerveau régule plusieurs hormones dont le cortisol, plus connue sous l'appellation d' « hormone du stress ». Lorsqu'activé, l'hypothalamus stimule l'hypophyse qui déclenche en retour la libération du cortisol par les glandes surrénales (Kemeny, 2003). Le cortisol ainsi relâché dans l'organisme a pour effet d'inhiber certaines fonctions pour permettre au corps de garder ses ressources afin de faire face au stimulus aversif. Lors d'un stress intense ou prolongé, l'effet du cortisol a des conséquences délétères pour l'individu et peut même inhiber le processus de guérison, la digestion et la croissance (Kemeny, 2003).

On retrouve de nombreux récepteurs au cortisol dans diverses structures cérébrales dont l'amygdale de l'axe HPA. Cette structure est constituée d'une paire de noyaux cérébraux appartenant au système limbique. Elle est impliquée dans la reconnaissance et l'évaluation de la valence émotionnelle des stimuli sensoriels, dans l'apprentissage associatif et dans les réponses comportementales et végétatives associées à la peur et à l'anxiété (Perry, 2001; Lotstra, 2002). L'amygdale fonctionne comme un détecteur et un système d'alarme pour reconnaître les sources de stress provenant de l'environnement. Par la suite, les stimuli menaçants seront encodés pour permettre à l'organisme de reconnaître rapidement cette information déclenchant la peur et l'anxiété afin de réagir immédiatement. Un stimulus pourra donc provoquer instantanément les réactions de peur et d'anxiété (voie courte) sans passer par un traitement cortical de l'information (voie longue) (circuit de la peur; LeDoux, 1994). Bien que l'amygdale joue un rôle important dans le maintien de la survie de l'individu, elle est la principale cause de la difficulté de régulation de l'anxiété, la voie courte étant devenue la voie de traitement préférentielle du cerveau face à certains stimuli normalement moins anxiogènes. En effet, l'amygdale intervient 
aussi dans le processus de mémorisation; elle ajoute une valence émotionnelle à l'information afin de favoriser la rétention. Elle est également impliquée dans le processus d'apprentissage par conditionnement classique aversif. L'étude de Phan, Fitzerald, Nathan et Tancer (2006) a démontré que chez les personnes souffrant d'anxiété sociale, l'hyperactivité de l'amygdale ainsi que l'ampleur de son activation seraient positivement associées à la sévérité de leurs symptômes. L'hyperactivité de l'amygdale provoquerait une émotivité intense pouvant aller jusqu'à la peur extrême qui caractérise les crises de panique, conduisant l'individu à fuir les situations provoquant cette hyperactivité.

Concernant les relations d'attachement, les traumas psychologiques précoces auraient un impact sur le développement de l'amygdale et le circuit de la peur pendant la maturation du cerveau. Ces perturbations deviendraient par la suite permanentes (Thomas et al., 2001). Ainsi, une étude effectuée par Lecompte, Moss, Cyr et Pascuzzo (2014) démontre que des préadolescents qui ont présenté un patron d'attachement désorganisé avant l'âge scolaire manifestent des symptômes d'anxiété et de dépression. D'autres études confirment également l'association d'un attachement désorganisé avec l'anxiété chez l'enfant (Kerns \& Brumariu, 2014; Kerns, Siener, \& Brumariu, 2011; Moss, Smolla, Guerra, Mazzarello, \& Chayer, 2006). Certains auteurs avancent que le TRA peut être considéré comme un cas de désorganisation extrême de la relation d'attachement (van IJzendoorn \& Bakermans-Kranenburg, 2003). Un enfant présentant un TRA serait donc plus disposé à souffrir d'anxiété. En effet, l'amygdale devenue hyperactive, puisque sur-stimulée lors des relations d'attachement précoces nonsécurisantes ou menaçantes, déclenche alors un traitement préférentiel des stimuli par « la voie courte », provoquant ainsi des difficultés de régulation de l'anxiété. Selon les critères mis de l'avant par le DSM-5 (APA, 2013), un enfant présentant un TRA démontre de la difficulté à se réconforter et trouver de la consolation lorsqu'il est en détresse et peut présenter des épisodes inexpliqués de peur dans des contextes relationnels non-menaçants. Les symptômes décrits semblent donc converger vers une hyperactivité de l'amygdale (voie courte) lors de relations interpersonnelles, et ce, de façon indifférenciée, celles-ci étant devenues un stimulus menaçant de par les expériences adverses précoces et non-sécurisantes vécues par l'enfant. Ainsi, l'activation fréquente de l'amygdale provoque une incapacité de l'enfant à réguler la peur et l'anxiété. En effet, le traitement rapide de la stimulation (voie courte) suite à l'activation de l'amygdale empêche le cerveau de procéder à une analyse plus raffinée du stimulus déclencheur de la peur et 
de l'anxiété. Cette analyse optimisée du stimulus effectuée par les structures corticales supérieures permettrait la modulation de l'action de l'amygdale sur les différentes structures responsables de l'expression corporelle de la peur telle que l'accélération du pouls, la pâleur, la sudation et l'immobilisation du corps (Lotstra, 2002).

Plusieurs études démontrent l'association entre un attachement de type insécure avec la présence de symptômes dépressifs durant l'enfance et la préadolescence (Armsden \& Grennberg, 1987; Kaplan \& Main, 1986; Lecompte et al., 2014). McGowan et al. (2009) observent, quant à eux, l'altération de l'axe HPA chez les victimes de suicide ayant vécu des expériences précoces adverses, contrairement à ceux n'ayant pas cet historique ou le groupe de contrôle. Plus particulièrement, l'étude de Fenoglio et al. (2006) démontre que, dès les premiers jours de vie, et suite à une augmentation des soins maternels, des modifications sont observées dans la modulation de la réponse au stress par la voie HPA et l'amygdale, modifications devenant permanentes par la suite. Ces résultats indiquent qu'une interaction maternelle de qualité médiocre induirait le développement d'un individu qui, à l'âge adulte, serait plus craintif et plus inquiet, réagissant de façon rapide et intense par le relâchement de cortisol aux situations de stress. En outre, chez de jeunes enfants exposés à des traumas répétitifs durant la guerre, la sécurité de l'attachement avec la mère est un facteur de résilience et de protection concernant l'apparition d'un trouble de stress post-traumatique chez ceux-ci (Feldman \& Vengrober, 2011). Dans le TRA, la perturbation de la qualité des soins prodigués par les figures significatives est un critère nécessaire au diagnostic, soit par de la négligence extrême, des changements répétés de figure de soins ou l'institutionnalisation de l'enfant. Les jeunes ayant un diagnostic de TRA seraient donc à risque de présenter une altération de l'axe HPA pouvant devenir permanente. Cette altération provoquerait des difficultés de régulation de l'anxiété et pourrait induire des éléments dépressifs ultérieurement. En effet, les critères diagnostiques du DSM-5 (APA, 2013) font mention d'épisodes de tristesse et de peu d'affects positifs chez les enfants présentant un TRA, manifestations permettant de supposer une perturbation du fonctionnement de l'axe HPA.

\section{L'hippocampe}

Dans leur étude, Fenoglio et al. (2006) observent que les individus ayant bénéficié de soins maternels médiocres développent préférentiellement une capacité d'apprentissage par évitement ainsi qu'une altération des processus d'apprentissage plus complexes comme la mémorisation, le contrôle attentionnel et les habiletés visuo-spatiales. Ces processus, ainsi que 
celui de l'inhibition du comportement, relèvent de l'hippocampe, structure dont le fonctionnement est inhibé par l'amygdale lors de production de cortisol en présence de stress. Cette désactivation a pour but de mobiliser les ressources de l'individu pour se battre ou s'enfuir. Son inhibition aura pour effet d'orienter l'attention et la disponibilité de l'individu vers la menace et non plus vers les apprentissages. L'excès de cortisol dans l'organisme ainsi que sa présence prolongée provoquera une atrophie de l'hippocampe, une réduction des capacités de rétention et d'apprentissage de la personne ainsi qu'un affaiblissement du système immunitaire (Lupien et al., 1998; Perry, 2001; Polan \& Hoffer, 2008). Dans une recension des études concernant la réponse de cortisol chez les enfants âgés entre 0 et 5 ans vivant dans des conditions adverses (p. ex., maladie mentale chez la mère, toxicomanie maternelle, mère abusive), Hunter, Minnis et Wilson (2011) rapportent une augmentation de la sécrétion de cortisol dans la majorité des études considérées. De même, les enfants vivant en institution présentent un patron de production diurne de cortisol atypique comparé aux enfants n'y résidant pas (p. ex., sans pic matinal) (Dozier et al., 2006). Un faible taux de cortisol a été associé à des comportements agressifs chez les enfants (McBurnett, Lahey, Rathouz, \& Loeber, 2000). Les recherches suggèrent donc que les conditions précoces adverses telles que les relations d'attachement perturbées interfèrent avec les mécanismes de régulation neuroendocrinien des enfants (p. ex., le cortisol), ce qui induit en retour des altérations dans le développement de plusieurs structures impliquées au niveau de la régulation émotionnelle et des processus cognitifs tels que l'apprentissage, le contrôle attentionnel et la mémorisation.

Au niveau cortical supérieur

L'amygdale et l'hippocampe étendent leurs connexions au niveau cortical supérieur permettant aux structures cérébrales corticales d'assurer leurs fonctions de régulation sur les comportements, les émotions et l'attention. Ces aires cérébrales, telles que la région orbitofrontale du cortex préfrontal, exercent une influence sur le système de recherche ou d'évitement des stimuli, de réponses conditionnées ou d'extinction de la réponse, ainsi que sur le contrôle cognitif de l'attention ou d'évaluation des stimuli. Ces régions préfrontales sont reliées aux processus d'attachement d'au moins deux façons. D'abord, elles sont impliquées dans l'encodage des réponses automatiques liées aux figures d'attachement concernant les réponses excitatrices ou inhibitrices face à des indices de menaces (région orbitale médiane). Ensuite, elles sont impliquées dans la modulation des opérations cognitives associées à l'attachement telles que les 
capacités réflexives et la mémoire de travail (région dorsolatérale) (Bretherton \& Munholland, 2008). Le cortex préfrontal est également le siège des fonctions exécutives regroupant les processus attentionnels, la mémoire de travail et le contrôle de l'impulsivité. De plus, au niveau orbito-frontal, se situent les sites dédiés à l'apprentissage des récompenses sensorielles et psychologiques tel que la valeur de la vue d'un visage, comme celui de son enfant pour sa mère par exemple (Bartels \& Zeki, 2004). Des dommages à la zone orbito-frontale du cortex préfrontal chez les jeunes enfants seraient liés à des difficultés à exprimer et expérimenter la sympathie et les émotions de gêne et de culpabilité. Ces enfants démontrent également de grandes difficultés à développer une compréhension des règles sociales et des violations de ses règles (Bretherton \& Munholland, 2008). De plus, l'encodage des représentations d'attachement et des MOI serait possible grâce au système des neurones miroir, système qui permet la simulation mentale des actions des autres, des buts et des émotions. Selon ces auteurs, des sites cérébraux sensibles à l'interprétation et l'imagination des interactions sociales sont situés dans les cortex temporal et préfrontal et l'altération de ces sites perturbera en retour le système des représentations d'attachement et des MOI.

Des altérations au niveau préfrontal auront donc des répercussions importantes sur les plans comportemental et social; les situations d'attachement fortement perturbées provoqueraient des atteintes également à ce niveau. En effet, l'étude de DeKlyen \& Greenberg (2008) a montré que le développement de problèmes de comportement chez les enfants d'âge scolaire est influencé par la qualité de l'attachement de l'enfant envers son parent. Ainsi, les enfants ayant un patron d'attachement insécure de type désorganisé et ambivalent présentent plus de problèmes de comportement extériorisés et intériorisés que les enfants ayant un attachement sécurisant (Moss et al., 2006). De même, Granot et Mayseless (2001) démontrent que des enfants âgés de 10 ans ayant des représentations d'attachement évitant ou désorganisé présentent plus de problèmes de comportement que des enfants ayant un l'attachement sécurisé. En outre, les enfants présentant un patron d'attachement insécure seraient moins susceptibles de développer des capacités de régulation des émotions efficaces et des habiletés d'interaction sociales adéquates (Kerns \& Brumariu, 2014; Kerns et al., 2011). D’autres études révèlent également une association positive entre une sécurité d'attachement précoce et la qualité des amitiés et des relations avec les pairs durant l'enfance (Berlin, Cassidy, \& Appleyard, 2008). La qualité de l'attachement influencerait donc la qualité mais aussi la quantité des amitiés chez des enfants d'âge scolaire (Elicker, 
Englund, \&Sroufe, 1992; Lewis \& Feiring, 1989). Il existerait également une association entre la sécurité de l'attachement (patron d'attachement sécure), avoir des relations plus harmonieuses avec les pairs, être mieux considérer de ceux-ci et démontrer moins de problèmes de comportement à l'âge scolaire (Berlin \& Cassidy, 1999; Thompson, 2008). Une importante étude longitudinale effectuée au Minnesota a démontré que l'attachement mère-enfant prédit les compétences avec les pairs 15 à 16 ans plus tard (Englund, Levy, Hyson, \& Sroufe, 2000; Sroufe, Egeland, \& Carlson, 1999). Par ailleurs, dans une étude utilisant l'imagerie cérébrale, Chugani et al. (2001) ont démontré une activité métabolique limitée dans les zones cérébrales frontales, préfrontales, temporales, au niveau de l'amygdale ainsi que de l'hippocampe chez des enfants des orphelinats roumains ayant vécu de multiples formes de négligence. De nombreux résultats indiquent donc des perturbations quant au développement et au fonctionnement des structures corticales supérieures correspondant aux symptômes du TRA décrits par le DSM-5 (APA, 2013), à savoir les réponses émotionnelles et sociales minimales envers les autres ainsi que des épisodes d'irritabilité lors de relations non-menaçantes avec des adultes.

Il est également reconnu que les enfants ayant un diagnostic de TRA présentent des difficultés d'apprentissage. Ainsi, Schwarz (2006) rapporte que les ressources de ces enfants sont essentiellement investies dans leur survie de par leur histoire de maltraitance et de changement fréquent de pourvoyeur de soins, les laissant incapables de bénéficier des apprentissages dispensés à l'école. Selon Damen et al. (2012), les enfants présentant un TRA démontrent des symptômes ressemblant à ceux du trouble déficitaire de l'attention avec hyperactivité (TDAH) pouvant s'expliquer par les connexions profondes entre les lobes frontaux et les structures du système limbique (axe HPA et amygdale en particulier). En effet, selon ces auteurs, la dysfonction limbique serait primaire au TDAH observé chez ces enfants, ce qui ne serait pas le cas des enfants présentant un TDAH sans TRA. L'étude de Kreppner, O’Connor et Rutter (2001) indique même que les symptômes d'inattention et d'hyperactivité sont liés positivement avec la sévérité des privations et des négligences subis par l'enfant. De plus, les enfants ayant vécu en institution présenteraient un développement anormal de substance blanche (tissu du système nerveux central composé de fibres nerveuses reliant les neurones entre eux) dans les lobes frontaux, temporaux et pariétaux; un impact qui serait associé avec la durée de la période d'institutionnalisation et les symptômes d'inattention et d'hyperactivité évalués en neuropsychologie (Govindan, Behen, Helder, Makki, \& Chugani, 2010). Certaines recherches 
suggèrent également une altération des capacités de mémorisation et d'apprentissage suite à l'atrophie de l'hippocampe induite par l'excès de cortisol secrété par l'organisme (Perry, 2001; Polan \& Hoffer, 2008).

Des perturbations au niveau du fonctionnement de diverses structures cérébrales seraient donc présentes chez l'enfant ayant reçu un diagnostic de TRA. Ces altérations provoqueraient des troubles à de nombreux niveaux touchant les comportements, la régulation émotionnelle, le contrôle attentionnel, les habiletés sociales et les apprentissages. Certaines de ces altérations pourraient même devenir permanentes et continuer d'affecter le fonctionnement de l'individu à l'âge adulte. La sécurité de l'attachement serait, en outre, un facteur de protection concernant certains troubles de santé mentale tels le trouble stress post-traumatique, laissant entrevoir la spécificité et l'importance des effets délétères de relations d'attachement perturbées par rapport à d'autres traumatismes pourtant sévères (p. ex., la guerre).

\section{CONCLUSION}

De nombreux résultats d'études mettent en lumière l'impact des relations d'attachement perturbées sur plusieurs sphères du développement chez l'enfant. En effet, les enfants présentant un TRA démontrent des difficultés importantes concernant la régulation émotionnelle et comportementale, le contrôle de l'attention, les apprentissages et les habiletés sociales. Actuellement, de par les avancés des recherches en neuroscience, l'évidence de l'influence des relations précoces perturbées sur le développement et le fonctionnement de certaines structures du cerveau émerge. En effet, des altérations sont relevées au niveau de l'axe HPA, système modulant la production et l'impact du cortisol sur le cerveau, ainsi que d'autres structures du système limbique telles que l'hippocampe et les structures corticales supérieures. L'enfant soumis continuellement à des situations stressantes et adverses sans possibilité d'être sécurisé par une figure d'attachement adéquate n'est pas en mesure de pouvoir réguler les systèmes activés lors de la présence de danger (axe HPA, plus particulièrement l'amygdale). La production accrue de cortisol et la sur-activation de ces systèmes sans possibilité d'autorégulation ont des effets délétères sur le développement et le fonctionnement de nombreuses structures cérébrales, effets pouvant même devenir permanents. En effet, dès les premiers jours de vie, l'impact de relations d'attachement perturbées interfère avec le fonctionnement du cycle de production de cortisol et de celui de l'axe HPA nuisant au développement d'un système de régulation des émotions adéquates (p. ex., anxiété, dépression). Ce débalancement se répercute sur d'autres structures 
impliquées dans les processus de mémorisation, d'apprentissage et des habiletés visuo-spatiales (hippocampe) ainsi qu'au niveau cortical supérieur, altérant les capacités attentionnelles, le contrôle des impulsions, la mémoire de travail, les capacités réflexives et les habiletés sociales (p. ex., régions préfrontales). Au niveau symptomatologique et clinique, ces atteintes peuvent se manifester chez l'enfant présentant un TRA par des difficultés de régulation émotionnelle (p. ex., anxiété, dépression), des troubles de comportement, des troubles de l'attention, des troubles d'apprentissage et des difficultés au niveau des habiletés sociales.

Dans cette optique, l'étude des profils d'enfants d'âge scolaire ayant reçu un diagnostic de TRA pourrait permettre d'effectuer un rapprochement entre les manifestations cliniques présentées et les atteintes cérébrales décrites par les recherches en neuroscience. Mieux comprendre les altérations profondes provoquées par les relations d'attachement perturbées favoriserait l'élaboration d'interventions plus ciblées et spécifiques ainsi que l'utilisation d'indicateurs biologiques pertinents (p. ex., le taux de cortisol). Depuis longtemps, les relations d'attachement précoces sont reconnues comme primordiales au développement de l'enfant. Actuellement, les études nous indiquent qu'elles sont le terreau nécessaire au bon développement de son cerveau. 


\section{Références}

Ainsworth, M. D., Blehar, M. C., Waters, E., \& Walls, S. (1978). Patterns of attachment: A psychological study of the strange situation. Oxford: Earlbaum.

American Psychiatry Association. (1980). Diagnostic and statistical manual of mental disorders ( $3^{\text {th }}$ edition revised). Arlington, VA: American Psychiatric Association Publishing.

American Psychiatry Association. (2000). Diagnostic and statistical manual of mental disorders ( $4^{\text {th }}$ edition revised). Arlington, VA: American Psychiatric Association Publishing.

American Psychiatry Association. (2013). Diagnostic and statistical manual of mental disorders ( $5^{\text {th }}$ edition). Arlington, VA: American Psychiatric Association Publishing.

Aoki, Y., Zeanah, C. H., Heller, S. S., \& Bakshi, S. (2002). Parent-infant relationship global assessment scale: A study of its predictive validity. Psychiatry and Clinical Neurosciences, 56(5), 493-497.

Armsden, G. C., \& Greenberg, M. T. (1987). The inventory of parent and peer attachment: Individual differences and their relationship to psychological well being in adolescent. Journal of Youth and Adolescence, 16, 427-454.

Bartels, A., \& Zeki, S. (2004). Functional brain during free viewing of natural scenes. Human Brain Mapping, 21(2), 75-85.

Berlin, L. J., \& Cassidy, J. (1999). Relations among relationships: contributions from attachment theory and research In J. Cassidy \& P. R. Shaver (Eds.), Handbook of attachment: Theory, research, and clinical applications ( $1^{\text {st }}$ ed., pp. 688-712). New York, NY: Guilford Press.

Berlin, L. J., Cassidy, J., \& Appleyard, K (2008). The influence of attachment security and related constructs in infancy and early childhood, In J. Cassidy \& P. R. Shaver (Eds.), Handbook of attachment: Theory, research, and clinical applications $\left(2^{\text {nd }}\right.$ ed., pp. 383416). New York, NY: Guilford Press.

Bowlby, J. (1969). Attachment and loss, vol.1. New York, NY: Basic Books.

Bowlby, J. (1973). Attachment and loss, vol.2: Separation. New York, NY: Basic Books.

Bowlby, J. (1980). Attachment and loss, vol.3: Loss, sadness and depression. New York, NY: Basic Books.

Borelli, J. L., David, D. H., Crowley, M. J., \& Mayes, L. C. (2010). Links between disorganized attachment classification and clinical symptoms in school-aged children. Journal of Child \& Family Studies, 19(3), 243-256. 
Boris, N. W., Fueyo, M., \& Zeanah, C. H. (1997). The clinical assessment of attachment in children under five. Journal of the American Academy of Child \& Adolescent Psychiatry, 36(2), 291-293.

Boris, N. W., Zeanah, C. H., Larrrieu, J. A., Scheeringa, M. S., \& Heller, S. (1998). Attachment disorders in infancy and early childhood: A preliminary investigation of diagnostic criteria. American Journal of Psychiatry, 155(2), 295-297.

Bretherton I., \& Munholland, K. A. (2008). Internal working model in attachment relationships: Elaborating a central construct in attachment theory. In J. Cassidy \& P. R. Shaver (Eds.), Handbook of attachment: Theory, research, and clinical applications $\left(2^{\text {nd }}\right.$ ed., pp. 102127). New York, NY: Guilford Press.

Bureau, J. F., Béliveau, M. -J., Moss, E., \& Lépine, S. (2006). Association entre l'attachement mère-enfant et les récits d'attachement à la période scolaire. Revue canadienne des sciences du comportement, 38(1), 50-62.

Bureau, J. -F., \& Moss E. (2010). Behavioral precursor of attachment representations in middle childhood and links with child social adaptation. British Journal of Developmental Psychology, 28(3), 657-677.

Carlson, E. A. (1998). A prospective longitudinal study of attachment disorganizationdisorientation. Child Development, 69(4), 1107-1128.

Chaffin, M., Hanson, R., Saunders, B. E., Nichols, T., Barnett, D., Zeanah, C., Berliner, L., Egeland, B., Newman, E., Lyon, T., Letourneau, E., \& Miller-Perrin, C. (2006). Report of the APSAC Task Force on attachment therapy, reactive attachment disorder, and attachment problems. Child Maltreatment, 11(4), 76-89.

Chugani, H. T., Behen, M. E., Muzik, O., Juhasz, C., Nagy, F., \& Chugani, D. C. (2001). Local brain functional activity following early deprivation: A study of postinstutitionalized Romanian orphans. NeuroImage, 14(6), 1290-1301.

Coleman, P. K. (2003). Reactive Attachment Disorder in the context of the family: A review and call for further research. Emotional and Behavioral Difficulties, 8(3), 205-216.

Crittenden, P. M. (1985). Maltreated infants: vulnerability and resilience. The Journal of Child Psychology and Psychiatry, 26(1), 85-96. 
Damen, B., Pütz, V., Herpetz-Dahlmann, B., \& Konrad, K. (2012). Early pathogenic care and the development of ADHD-like symptoms. Journal of Neural Transmission, 119(9), 10231036.

DeKlyen, M., \& Greenberg, M. T. (2008). Attachment and psychopathology in childhood. In J. Cassidy \& P. R. Shaver (Eds.), Handbook of attachment: Theory, research, and clinical applications ( $2^{\text {nd }}$ ed., pp. 637-665). New York, NY: Guilford Press.

Dozier, M., Manni, M., Gordon, M. K., Peloso, E., Gunnar, M. R., Stovall-McClough, K. C., Eldreth, D., \& Levine, S. (2006). Foster children's diurnal production of cortisol: An exploratory study. Child Maltreatment, 11(2), 189-107.

Egeland, B., \& Carlson, E. (2004). Attachment and psychopathology. In L. Atkinson \& S. Goldberg (Eds.), Attachment issues in psychopathology and intervention (pp. 27-48). Mahwah, NJ : Lawrence Erlbaum Associates Publishers.

Englund, M. M., Levy, A. K., Hyson, D. M., \& Sroufe, L. A. (2000). Adolescent social competence: Effectiveness in a group setting. Child Development, 71(4), 1049-1060.

Elicker, J., Englund, M., \& Sroufe, L. A. (1992). Predicting peer competence and peer relationships in childhood from early parent-child relationships. In R. D. Parke \& G. W. Ladd (Eds.), Family-peer relationshilps: mode of linkage (pp. 77-106). Hillsdale, NJ : Lawrence Erlbaum Associates.

Fearon, R. P., Bakermans-Kranenburg, M. J., van IJzendoorn, M. H., Lapsley, A. M., \& Roisman, G. I. (2010). The significance of insecure attachment and disorganization in the development of children's externalizing behavior: a meta-analytic study. Child Development, 81(2), 435-456.

Fenoglio, K. A., Chen, Y., \& Baram, T. Z. (2006). Neuroplasticity of the hypothalamic-pituitaryadrenal axis early life requires recurrent recruitment of stress-regulating brain regions. The Journal of Neuroscience, 26(9), 2434, 2442.

Gleason, M. M., Fox, N. A., Drury, S., Smyke, A., Egger, H. L., Nelson III, C. A., Gregas, M. C., \& Zeanah, C. H. (2011). Validity of evidence-derived criteria for Reactive Attachment Disorder: Indiscriminately social/disinhibited and emotionally withdrawn/inhibited types. Journal of American Academy of Child and Adolescent Psychiatry, 50(3), 216-231. 
Gleason, M. M., \& Zeanah, C. H. (2010). Assessing infants and toddlers. In M. K. Dulcan (Ed.), Dulcan's textbook of child and adolescent psychiatry (pp. 3-14). Arlington, VA: American Psychiatry Publishing.

Govindan, R. M., Behen, M. E., Helder, E. Makki, M. I., \& Chugani, H. T. (2010). Altered water diffusy in cortical association tracts in children with early deprivation identification with tract-based spatial statistics (TBSS). Cerebral Cortex, 20(2), 561-569.

Granot, D., \& Mayseless, O. (2001). Attachment security and ajustement to school in middle childhood. International Journal of Behavioral Development, 25(6), 530-541.

Green, J., \& Goldwyn, R. (2002). Annotation: Attachment disorganization and psychopathology : New findings in attachment research and their potential implications for developmental psychopathology in childhood. Journal of Child Psychology and Psychiatry, 43(7), 835846.

Hanson, R. F., \& Spratt, E. G. (2000). Reactive Attachment Disorder: What we know about the disorder and implications for the treatment. Child Maltreatment, 5(2), 137-145.

Hunter, A. L., Minnis, H., \& Wilson, P. (2011). Altered stress responses in children exposed to early adversity: A systematic review of salivary cortisol studies. The International Journal on the Biology of the Stress, 14(6), 614-626.

Kaplan, N., \& Main, M. (1986). Instructions for the classification of children's family drawings in terms of representation of attachment. Berkeley, CA: University of California.

Kemeny, M. E. (2003). Psychology of stress. Current Directions in Psychology Science, 12(4), 124-129.

Kerns, K. A., \& Brumariu, L. E. (2014). Is insecure parent-child attachment a risk factor for the development of anxiety in childhood or adolescence? Child Development Perspectives, $8(1), 12-17$.

Kerns, K. A., Siener, S., \& Brumariu, L. E. (2011). Mother-child relationships, family context, and child characteristics as predictors of anxiety symptoms in middle childhood. Development and Psychopathology, 23(2), 593-604.

Kreppner, J. M, O’Connor, T. G., \& Rutter, M. (2001). Can inattention/overactivity be an institutional deprivation syndrome? Journal of Abnormal Child Psychology, 29(6), 513528. 
Lecompte, V., Moss, E., Cyr, C., \& Pascuzzo, K. (2014). Preschool attachment, self-esteem and the development of preadolescent anxiety and depressive symptoms. Attachment \& Human development, 16(3), 242-260.

LeDoux, J. E. (1992). Emotion and the amygdale In J. P. Aggleton (Ed.), The amygdale: Neurobiological aspects of emotion, memory, and mental dysfunction (pp. 339-351). Wiley-Liss, NY: Wiley.

Lewis, M., \& Feiring, C. (1989). Infant, mother, and infant-mother interaction behavior and subsequent attachment. Child Development, 60(4), 831-837.

Lotstra, F. (2002). Le cerveau émotionnel ou la neuroanatomie des émotions. Cahiers critiques de thérapie familiale et de pratiques de réseaux, 29(2), 73-86.

Ludy-Dobson, C., \& Perry B. D. (2010). The role of healthy relational interaction in buffering the impact of childhood trauma. In E. Gilwith (Ed.), Working with children to heal interpersonal trauma: The power of play (pp. 26-42). New York, NY: Guilford Press.

Lupien, S. J., de Leon, M., de Santi, S., Convit, A., Tarshi, C., Nair, N. P. V., Thakur, M., McEwen, B. S., Hauger, R. L., \& Meaney, M. J. (1998). Cortisol levels during human aging predict hippocampal atrophy and memory deficits. Nature Neuroscience, 1(4), 6973.

Lyons-Ruth, K. (1996). Attachment relationships among children with aggressive behavior problems: The role of disorganized early attachment patterns. Journal of Consulting and Clinical Psychology, 64(1), 64-73.

Lyons-Ruth, K., Alpern, L., \& Repacholi, B. (1993). Disorganized infant attachment classification and maternal psychosocial problems as predictors of hostile-aggressive behavior in the preschool classroom. Child Development, 64(2), 572-585.

Main, M. (1998). De l'attachement à la psychopathologie. Enfance, 51(3), 13-27.

Main, M., \& Hesse, E. (1990). Parent's unresolved traumatic experiences are related to infant disorganized attachment status: Is frigntened and/or frightened parental behavior the linking mechanism? In M. T. Greenberg, D. Cicchetti \& E. M. Cummings (Eds.), Attachment in the preschool years: Theory, research, and intervention (pp. 121-160). Chicago, IL: University of Chicago Press. 
Main, M., Kaplan, N., \& Cassidy, J. (1985). Security in infancy, childhood, and adulthood: a move to the level of representation. Monographs of the Society for Research in Child Development, 50(1-2), 66-104.

Main, M., \& Solomon, J. (1990). Procedures for identifying infants as disorganised/disoriented during the Ainsworth's Strange Situation. In M. T. Greenberg, D. Cicchetti \& E. M. Cummings (Eds.), Attachment in the preschool years: Theory, research, and intervention (pp. 121-160). Chicago, IL: University of Chicago Press.

McBurnett, K., Lahey, B. B., Rathouz, P. J., \& Loeber, R. (2000). Low salivary cortisol and persistent aggression in boys referred for disruptive behavior. Archives of General Psychiatry, 57(1), 38-43.

McGowan, P. O., Sasaki, A., D’Alessio, A. C., Dymov, S., Labonté, B., Szyf, M., Turecki, G., \& Meany, M. J. (2009). Epigenetic regulation of the glucocorticoid receptor in human brain associates with childhood abuse. Nature Neuroscience, 12(3), 342-348.

Minnis, H., Marwick, H, Arthur, J., \& McLaughlin, A. (2006). Reactive Attachment Disorder: A theoretical model beyond attachment. European Child \& Adolescent Psychiatry, 15(6), 336-342.

Moss, E., Smolla, N., Guerra, I., Mazzarello, T., \& Chayer, D. (2006). Attachement et problèmes de comportements intériorisés et extériorisés auto-rapportés à la période scolaire. Canadian Journal of Behavioral Science, 38(2), 142-157.

Newman, L., \& Mares S. (2007). Recent advances in the theories of and interventions with attachment disorders. Child and adolescent psychiatry, 20 (4), 343-348.

O'Connor, T. G., \& Zeanah C. H. (2003). Current perspectives on attachment disorders: Rejoinder and synthesis. Attachment \& Human Development, 5(3), 321-326.

Phan, K. L., Fitzerald, D. A., Nathan, P. J., \& Tancer, M. E. (2006). Association between amygdale hyperactivity to harsh faces, and severity of social anxiety in generalized social phobia. Biological Psychiatry, 59(5), 424-429.

Perry, B. D. (2001). The neurodevelopmental impact of violence in childhood In D. Schetky \& E. P. Benedek (Eds.), Textbook of child and adolescent forensic psychiatry (pp. 221-238). Arlington, VA: American Psychiatric Press. 
Perry, B. D. (2008). Child maltreatment: The role of abuse and neglect in developmental psychopathology. In T. P. Beauchaine \& S. P. Hinshaw (Eds.), Textbook of child and adolescent psychopathology (pp. 93-128). Wiley-Liss, NY: Wiley.

Polan, H. J., \& Hofer, M. R. (2008). Psychobiological origins of infant attachment and its role in development In J. Cassidy \& P. R. Shaver (Eds.), Handbook of attachment: Theory, research, and clinical applications ( $2^{\text {nd }}$ ed., pp. 158-172). New York, NY: Guilford Press.

Rutter, M., Kreppner, J., \& Sonuga-Barke, E. (2009). Emanuel Miller Lecture: Attachment insecurity, disinhibited attachment, and attachment disorders: Where does research findings leave the concepts. The Journal of Child Psychology and Psychiatry, 50(5), 529543.

Schwarz, E. (2006). Reactive Attachment Disorder: Implications for school readiness and school functioning. Psychology in the School, 43(4), 471-479.

Smyke, A. T., Dumitrescu, A., \& Zeanah, C. H. (2002). Attachment disturbances in young children, I: The continuum of caretaking casuality. Journal of American Academic Child and Adolescent Psychiatry, 41(8), 972-982.

Spitz, R. (1946). Anaclitic depression: an inquiry into the genesis of psychiatric conditions in early childhood. Psychoanalytic Study of the Child, 1, 47-53.

Sroufe, L. A., Egeland, B., \& Carlson, E. A. (1999). One social world: The integrated development of parent-child and peer relationships. In W. A. Collins \& B. Laursen (Eds.), Relationships as developmental contexts (pp. 241-261). Mahwah, NJ: Lawrence Erlbaum Associated Associates Publishers.

Sroufe, L. A., Egeland, B., Carlson, E., \& Collins, W. A. (2005). Placing early attachment experiences in developmental context: The Minnesota longitudinal study. In K. E. Grossman, K. Grossman \& E. Waters (Eds.), Attachment from infancy to adulthood: The major longitudinal studies (pp. 48-70). New York, NY: Guilford Press.

Thomas, K. M., Drevets, W. C., Dahl, R. E., Ryan, N. D., Birmaher, B., E., Clayton, H., D., Whalen, P. J., \& Casey, B. J. (2001). Amygdala response to fearful faces in anxious and depressed children. Archives of General Psychiatry, 58(11), 1057-1063.

Thompson, R. A. (2008). Early attachment and later development. Familiar questions, new answers. In J. Cassidy \& P. R. Shaver (Eds.), Handbook of attachment: Theory, research, and clinical applications ( $2^{\text {nd }}$ ed., pp. 348-365). New York, NY: Guilford Press. 
Tizard, B., \& Rees, J. (1975). The effect of early institutional rearing on the behavior problems and affectional relationships of four year-old children. The Journal of Child Psychology and Psychiatry, 16(1), 61-73.

van IJzendoorn, M. H. \& Bakermans-Kranenburg, M. J. (2003). Attachment disorders and disorganised attachment: Similar and different. Attachment \& Human Development, 5(3), 313-320.

van IJzendoorn, M. H., \& Kroonneberg, P. M. (1988). Cross-cultural patterns of attachment: A meta-analysis of the strange situation. Child Development, 59(1), 147-156.

van IJzendoorn, M. H., Schuengel, C., \& Bakermans-Kranenburg, M. J. (1999). Disorganized attachment in early childhood: Meta-analysis of precursors, concomitants, and sequelae. Development and Psychopathology, 11(2), 225-249.

Zeanah, C. H., Boris, N. W., \& Larrieu, J. A. (1997). Infant development and developmental risk: A review of the past 10 Years. Journal of the American Academy of Child \& Adolescent Psychiatry, 36(2), 165-178.

Zeanah, C., H., Boris, N. W., \& Lieberman, A. F. (2000). Attachment disorder in infancy. In A. J. Sameroff, M. Lewis \& S. M. Miller (Eds.), Handbook of developmental psychopathology ( $2^{\text {nd }}$ ed., pp. 293-307). New York, NY: Springer.

Zeanah, C. H., Boris, N. W., \& Scheeringa, M. S. (1997). Psychopathology in infancy. Journal Child Psychology \& Psychiatry \& Allied Disciplines, 38(1), 81-99.

Zero to three. (1994). DC: 0-3R: Diagnostic Classification of Mental Health and Developmental Disorders of Infancy and Early Childhood. Zero to Three/National Center for Infants, Toddlers and families.

Zero to three. (2005). DC: 0-3R: Diagnostic Classification of Mental Health and Developmental Disorders of Infancy and Early Childhood. (Ed. Rev.). Zero to Three/National Center for Infants, Toddlers and families. 
Introduction au second article 
Le premier article tente de préciser les prédictions des recherches en neurosciences quant aux impacts des relations d'attachement perturbées sur le développement et le fonctionnement cérébral des enfants. Le second article, quant à lui, explore les manifestations cliniques d'enfants présentant un diagnostic de TRA. La marginalité de cette clientèle ainsi que les critères d'admission à l'étude voulant que les enfants aient vécu avec la figure d'attachement principale durant les premiers 18 mois de vie ont réduit le nombre de cas disponible pour cette recherche. Cette particularité de la population étudiée a motivé également le choix d'une analyse de cas. En effet, l'analyse de cas permet une exploration plus approfondie des caractéristiques des enfants, ce qui correspond également à la visée exploratoire de la question à l'étude, à savoir si les résultats avancés par les recherches en neurosciences peuvent s'avérer utiles sur le plan clinique. Cette analyse approfondie de trois cas d'enfants présentant un TRA permettra de jeter les bases d'une réflexion concernant les possibles impacts neurodéveloppementaux d'un trouble d'attachement, impacts pouvant interférer sur plusieurs sphères du fonctionnement psychologique de l'enfant et se chroniciser, comme le laisse entrevoir le premier article.

Les trois enfants et familles participant à la recherche reçoivent des services en pédopsychiatrie dans un hôpital situé dans la banlieue de Montréal. Ils ont accepté de participer à une étude plus large concernant les représentations des enfants présentant un diagnostic de TRA et la capacité de mentalisation de leurs mères. Les enfants ont été référés suite au diagnostic de TRA reçu dans le service de pédopsychiatrie. Les familles participantes ont d'abord été contactées par les chercheurs. Les informations concernant le processus de recherche leur ont été transmises afin d'éclairer leur décision. Le formulaire d'information et de consentement, approuvé par le Comités d'éthique de la recherche (CÉR) de l'hôpital et Lettres et sciences humaines de l'Université de Sherbrooke, a été alors signé par les mères des enfants participant à 
l'étude. Les mères autorisaient également l'accès au dossier pédopsychiatrique de l'enfant dans le but de recenser l'histoire clinique ainsi que les divers diagnostics reçus par ceux-ci. Lors de la rencontre d'évaluation de la recherche, pendant que les enfants procédaient aux récits d'attachement (ASCT) avec un assistant de recherche, les mères complétaient l'inventaire de comportement $(\mathrm{CBCL})$ dans une autre salle. Les récits d'attachement recueillis auprès des enfants ont été analysés par des évaluateurs accrédités à faire la cotation du ASCT.

L'analyse des résultats a permis de relever des perturbations au niveau de la régulation du comportement, des émotions, de l'attention, ainsi que des difficultés et des retards d'apprentissage. Comme décrit dans le premier article, ces manifestations peuvent effectivement suggérer des altérations au niveau du fonctionnement de certaines structures cérébrales pouvant être affectées par la présence des conditions de vie menant au développement d'un TRA chez l'individu (Chugani et al., 2001). En effet, les symptômes au niveau de la régulation des émotions suggèrent un dysfonctionnement du système limbique et plus précisément de l'axe HPA (Fenoglio et al., 2006; Kemeny, 2003; Thomas et al., 2001). Ce dysfonctionnement peut se répercuter également sur les capacités de mémorisation, d'apprentissage et d'attention, ainsi que sur les habiletés sociales de l'enfant, et avoir un impact sur le fonctionnement de structures cérébrales telles que l'hippocampe et le cortex frontal (Bretherton \& Munholland, 2008; Chugani et al., 2001; Fenoglio et al., 2006). L'analyse des cas cliniques permet donc d'amorcer un rapprochement entre les connaissances issues des recherches en neuroscience et la symptomatologie observée chez les enfants présentant un diagnostic de TRA, ainsi que d'ouvrir la voie à de nouvelles avenues de recherche. 
Deuxième article 


\title{
CONVERGENCE ENTRE CLINIQUE ET NEUROSCIENCES CHEZ LE TROUBLE RÉACTIONNEL DE L'ATTACHEMENT : ILLUSTRATIONS CLINIQUES
}

\author{
Véronique NEUVILLE \\ Commission scolaire Marie-Victorin \\ Miguel M. TERRADAS \\ Guadalupe PUENTES-NEUMAN \\ Université de Sherbrooke \\ Claude GRENIER \\ Hôpital Charles LeMoyne
}

\section{CONVERGENCE BETWEEN CLINICAL OBSERVATIONS AND NEUROSCIENCES WITHIN THE REACTIVE ATTACHMENT DISORDER: CLINICAL ILLUSTRATIONS}

\author{
Véronique NEUVILLE, D.Ps., psychologue \\ Commission scolaire Marie-Victorin \\ Miguel M. TERRADAS, Ph.D., psychologue clinicien \\ Professeur \\ Département de psychologie \\ Université de Sherbrooke - Campus de Longueuil \\ Guadalupe PUENTES-NEUMAN, Ph.D., psychologue \\ Professeur \\ Département de psychologie \\ Université de Sherbrooke - Campus de Longueuil \\ Claude Grenier, M.D., pédopsychiatre \\ Hôpital Charles LeMoyne
}




\title{
CONVERGENCE ENTRE CLINIQUE ET NEUROSCIENCES CHEZ LE TROUBLE RÉACTIONNEL DE L'ATTACHEMENT : ILLUSTRATIONS CLINIQUES
}

\begin{abstract}
Résumé
Cette analyse de cas clinique a pour objectif de décrire le fonctionnement psychologique de trois enfants présentant un trouble réactionnel de l'attachement et de l'interpréter selon les connaissances sur les atteintes neurocognitives pouvant y être liées. Les difficultés émotionnelles et comportementales, les représentations d'attachement et les données issues des dossiers des enfants sont d'abord présentées. Des facteurs communs aux plans émotionnel, comportemental, des apprentissages et des représentations d'attachement ont été constatés. Leurs problèmes quant à la régulation des affects, de l'attention et des relations ainsi que leurs difficultés des apprentissages concordent avec le fonctionnement cérébral prédit par les recherches en neurosciences.
\end{abstract}

Mots-clés : Trouble réactionnel de l'attachement, neurosciences, psychopathologie

\begin{abstract}
This case analysis aims to describe the clinical manifestations of three children with a diagnosis of Reactive Attachment Disorder and interpret them in the light of knowledge on possible neurocognitive impairment related to this disorder. The emotional and behavioral difficulties, the attachment representations, and the clinical history of the children are first presented. Some common factors were found between these three cases in terms of emotional, behavioural, and learning difficulties as well as attachment representations. Their problems regarding affect, attention, and relational regulation, and their learning difficulties are consistent with the brain function predicted by research in neurosciences.
\end{abstract}

Key words: Reactive Attachment Disorder, neurosciences, psychopathology 
Dans un article précédent, Neuville, Puentes-Neuman et Terradas (sous presse) abordent l'impact du trouble réactionnel de l'attachement (TRA) sur le développement cérébral de l'enfant dans une tentative d'intégration des résultats de recherches en neurosciences à la compréhension de ce trouble. Le diagnostic de TRA, tel que décrit dans la cinquième édition du Manuel diagnostique et statistique des troubles mentaux (DSM-5; American Psychiatric Association [APA], 2013), désigne un enfant présentant une incapacité persistante à s'engager dans des interactions sociales avec des adultes lui procurant des soins ou à y répondre d'une manière appropriée au stade du développement (comportements excessivement inhibés, hypervigilants ou nettement ambivalents et contradictoires face aux pourvoyeurs de soin). Les enfants présentant un TRA démontrent des difficultés importantes concernant la régulation émotionnelle et comportementale, le contrôle de l'attention, les apprentissages et les habiletés sociales (Millward, Kennedy, Towlson, \& Minnis, 2006). Cependant, très peu d'études tentent d'effectuer un rapprochement entre les atteintes observées chez l'enfant ayant reçu un diagnostic de TRA et celles imputables à une altération des structures cérébrales pouvant être liées à cette psychopathologie. En se basant sur les recherches existantes en neurosciences, il a été possible de dégager des atteintes probables (Neuville et al., sous presse), mais le lien entre le TRA et ces altérations n'a pas encore été étudié ni démontré. Cette analyse de cas tente donc de décrire les manifestations cliniques de trois enfants d'âge scolaire ayant reçu un diagnostic de TRA et de les interpréter à la lumière des connaissances actuelles en neurosciences. L'objectif est d'apporter un éclairage différent à la compréhension de cette psychopathologie et d'amorcer une réflexion concernant les atteintes neurocognitives possibles du TRA.

\section{LES MÉCANISMES DU STRESS ET LA RÉACTION FACE AUX SITUATIONS TRAUMATIQUES}

II est actuellement reconnu que la production accrue de cortisol et la sur-activation de l'axe hypothalamo-hypophyso-surrénalien (axe HPA ou axe corticotrope), système modulant la production et l'impact du cortisol sur le cerveau, ont des effets délétères sur le développement et le fonctionnement de nombreuses structures cérébrales pouvant même devenir permanentes. Dans leurs études, Klasseens et al. (2009) et Klasseens (2010) démontrent que l'exposition à un ou des traumas durant l'enfance (p. ex., négligence, abus physiques et sexuels) est associée à une altération de la régulation du taux de cortisol par l'axe HPA, ce qui n'est pas observé chez les individus ayant vécu des traumas à l'âge adulte en 
l'absence d'expériences traumatiques durant l'enfance. Cette altération pourrait même devenir chronique et expliquer en partie la vulnérabilité face aux troubles de santé mentale chez l'adulte (p. ex., syndrome de stress post-traumatique). La sur-activation de l'axe HPA, de par ses connexions avec les autres systèmes cérébraux tel que l'hippocampe, provoque des perturbations au niveau des processus d'apprentissage comme la mémorisation, le contrôle attentionnel et les habiletés visuo-spatiales. Ces processus relevant de l'hippocampe seraient effectivement altérés chez les individus ayant bénéficiés de soins maternels inadéquats (Fenoglio, Chen, \& Baram, 2006). Des modifications de l'hippocampe sont également observées lors de stress intenses et prolongés (Perry, 2001; Pinto et al., 2015; Polan \& Hoffer, 2008). Au niveau cortical supérieur, soit les régions du cortex frontal, préfrontal et temporal, une activité métabolique limitée ainsi qu'un développement anormal de la substance blanche sont rapportés chez les enfants institutionnalisés ayant vécu de multiples formes de négligence (Chugani et al., 2001; Govindan, Behen, Helder, Makki, \& Chugani, 2010). Sur le plan comportemental, un fonctionnement anormal des zones frontales du cerveau aura un effet majeur sur de nombreux processus régulant les comportements, les apprentissages, l'expression des émotions et les habiletés sociales puisque ces zones sont impliquées dans la modulation des capacités réflexives, la mémoire de travail (Bretherton \& Munholland, 2008), le contrôle de l'attention (Lavie \& de Fockert, 2006), l'inhibition motrice, la réorientation et la mise à jour des comportements (Levy \& Wagner, 2011), ainsi que dans l'expression de la sympathie, la gêne, la culpabilité et la compréhension et le respect des règles sociales (Anderson, Bechara, Damasio, Tranel, \& Damasio, 1999) (voir Figure 1). Les relations précoces d'attachement sont donc déterminantes pour permettre le bon développement des capacités de régulation affective et comportementale en favorisant le maintien d'un état propice aux apprentissages et au bon développement cérébral (Ludy-Dobson \& Perry, 2010).

Insérer ici la Figure 1

\section{LES AUTRES MANIFESTATIONS CLINIQUES DU TRA}

Sur le plan clinique, l'enfant présentant un TRA peut également manifester des difficultés de régulation émotionnelle (p. ex., anxiété, dépression), des troubles de comportement, de l'attention et des apprentissages, ainsi que des difficultés au niveau des habiletés sociales. La présence de symptômes dépressifs chez les enfants ayant reçu un diagnostic de TRA a été aussi documentée (Millward et al. 
2006). Ces autres symptômes comorbides expliquent pourquoi le TRA continue encore actuellement d'être un sujet controversé. Pour van IJzendoorn \& Bakermans-Kranenburg (2003), il ressemble aux comportements des enfants ayant développé un patron d'attachement désorganisé. Pour d'autres auteurs, il constitue un indice d'un attachement non-formé (ou d'un non-attachement), l'enfant n'ayant pas eu accès à une figure de soin stable (Gleason \& Zeanah, 2010; Zeanah, Smyke, Koga, \& Carlson, 2005). II est reconnu actuellement que les perturbations dans les relations d'attachement entraînent des altérations dans les fondements de certains acquis développementaux, comme la régulation émotionnelle, pouvant alors mener vers une psychopathologie ultérieure (Egeland \& Carlson, 2004). La désorganisation de l'attachement demeure cependant distincte du trouble d'attachement, mais est considérée comme un facteur de risque concernant ce trouble (van IJzendoorn \& Bakermans-Kranenburg, 2003).

\section{LE TRA ET LES CLASSIFICATIONS D’ATTACHEMENT ISSUES DE LA PSYCHOLOGIE}

\section{DÉVELOPPEMENTALE}

Les expériences précoces avec les principales figures d'attachement permettent l'émergence progressive d'un système de pensées, de croyances, de souvenirs, d'attentes, d'émotions et de comportements concernant l'individu lui-même et les autres. Ces schèmes relationnels, nommés modèles opérants internes $(\mathrm{MOI})$, régulent, interprètent et prédisent les comportements lors des relations avec autrui et en particulier, avec les figures d'attachement (Bureau, Béliveau, Moss, \& Lépine, 2006; Main, Kaplan, \& Cassidy, 1985). Selon DeKlyen et Greenberg (2008), un attachement insécure aurait un impact important sur la future mésadaptation de l'individu de par la cristallisation des $\mathrm{MOI}$ caractérisés par de la colère, de la méfiance et de l'anxiété. Bien qu'il soit difficile d'établir une correspondance entre le TRA et les patrons d'attachement traditionnellement décrits en psychologie du développement (Coleman, 2003; Minnis, Marwick, Arthur, \& McLaughlin, 2006; Newman \& Mares, 2007; O’Connor \& Zeanah, 2003), l'étude effectuée par Zeanah et al. (2005) suggère que dans une population d'enfants roumains institutionnalisés le TRA est associé au degré de formation du lien d'attachement plutôt qu'à l'organisation d'un type particulier d'attachement. Peu de recherches décrivent cependant les représentations d'attachement caractérisant les enfants ayant reçu ce diagnostic. Ces représentations, accessibles par le biais des narratifs d'attachement chez l'enfant d'âge scolaire, permettent d'entrevoir comment se construit 
le monde affectif et cognitif de celui-ci sur lequel repose la régulation des comportements (Mayseless, 2005; Moss, Bureau, Béliveau, Zdebik, \& Lépine, 2009).

\section{FONCTIONNEMENT NEUROBIOLOGIQUE DE L'ENFANT AYANT REÇU UN DIAGNOSTIC DE TRA : \\ COMPRÉHENSION THÉORIQUE ET CLINIQUE}

La documentation scientifique nous permet de supposer la présence d'altérations au niveau des systèmes cérébraux responsables de la gestion du stress et de l'anxiété chez l'enfant présentant un TRA tel que l'axe HPA. Ce système module la production et l'impact du cortisol sur le cerveau, ainsi que le fonctionnement d'autres structures du système limbique telles que l'hippocampe et les structures corticales supérieures. L'enfant atteint d'un TRA, soumis constamment à des situations stressantes, ne pourra pas obtenir du réconfort et de la sécurité auprès de ses figures principales de soins, conditions indispensables pour lui permettre de réguler l'activité des systèmes stimulés en présence de danger (axe HPA, plus particulièrement l'amygdale). La production accrue de cortisol aura un effet délétère sur le fonctionnement de l'hippocampe, responsable de processus tels que la mémorisation, le contrôle attentionnel et les habiletés visuo-spatiales. Elle aura également un impact sur les structures corticales supérieures notamment le cortex frontal, responsable de la régulation des comportements, des apprentissages, de l'expression des émotions et des habiletés sociales. L'analyse de cas d'enfants ayant reçu un diagnostic de TRA pourrait donc permettre, à la lumière de leur symptomatologie, d'inférer des altérations des systèmes cérébraux concernés, à savoir l'axe HPA et les structures corticales supérieurs tel que le cortex frontal.

\section{OBJECTIF ET MÉTHODE DE L’ANALYSE DE CAS CLINIQUES}

Cet article propose d'étudier le profil d'enfants ayant reçu un diagnostic de TRA afin d'effectuer un rapprochement entre leurs manifestations cliniques et d'éventuelles perturbations neurodéveloppementales tel que prédit par les recherches en neurosciences. Les cas de trois enfants d'âge scolaire présentant un diagnostic de TRA sont présentés. Ils ont participé à une large étude concernant les représentations d'attachement chez l'enfant présentant un TRA menée dans le service de pédopsychiatrie d'un hôpital situé dans la banlieue de Montréal. Les trois enfants concernés résident avec leurs mères biologiques (dont un avec ses deux parents biologiques). Ils ont reçu les services de la protection de la jeunesse et séjourné en unité d'hospitalisation en pédopsychiatrie suite à d'importants 
troubles du comportement affectant leur fonctionnement autant dans le milieu familial que scolaire. L'étude a reçu l'approbation des comités d'éthique de la recherche de l'hôpital et de l'Université de Sherbrooke. Les consentements à la participation à l'étude obtenus, les mères biologiques ont fourni diverses informations, accepté l'accès au dossier pédopsychiatrique et rempli un questionnaire concernant les comportements de leurs enfants. Des données au sujet des représentations d'attachement ont été recueillies auprès de ces derniers.

Malgré le manque de correspondance entre le diagnostic de TRA et la qualité de la relation d'attachement vécue par l'enfant avec ses parents (Coleman, 2003; Minnis et al., 2006; Newman \& Mares, 2007; O'Connor \& Zeanah, 2003; Zeanah et al., 2005), il demeure important de comprendre le fonctionnement affectif des enfants portant ce diagnostic. La nature relationnelle des difficultés associées au TRA justifie une analyse approfondie de la qualité du lien parent-enfant. Ainsi, nous avons relevé les représentations d'attachement des enfants qui sont présentées en fonction de trois critères: les représentations relatives à la relation avec la mère, celles concernant le lien avec le père et les habiletés de l'enfant à reconnaître et réguler les émotions négatives.

Les représentations d'attachement des enfants ont été explorées en utilisant les Histoires d'attachement à compléter (Attachment Story Completion Task, ASCT; Bretherton, Ridgeway, \& Cassidy, 1990). À l'aide de figurines, les enfants ont été invités à compléter des histoires dont la mise en situation réfère à des thèmes anxiogènes en lien avec la relation aux parents. II est ainsi voulu que le système d'attachement soit activé. Les histoires produites par l'enfant sont réputées être guidées par ses représentations de la relation d'attachement. Ainsi, face à une situation nécessitant le soutien ou la protection de l'adulte, l'enfant produira une histoire qui rend compte de ce qu'il peut lui-même attendre dans le contexte de sa relation avec la figure d'attachement. En effet, selon plusieurs auteurs, les représentations d'attachement identifiées lors des histoires élaborées par l'enfant sont conformes avec les relations qu'il entretient avec ses parents en dehors des récits (Grych, Wachsmuth-Schlaefer, \& Klockow, 2002; Oppenheim, 2006; Steele et al., 2003). Ces recherches utilisaient cependant un système de cotation différent de celui employé dans la présente analyse de cas cliniques.

Les récits des enfants ont été ensuite analysés selon le Système de cotation centré sur l'attachement pour les histoires à compléter (Attachment Focused Coding System, AFCS; Reiner \& Splaun, 2008; 
Splaun, 2012). L'AFCS permet, à travers l'analyse de neuf échelles, d'identifier les représentations que l'enfant a de chacun de ses parents. Plus précisément, ce système de cotation s'intéresse aux représentations des parents comme étant soutenants ou rejetants, à la façon qu'a l'enfant de se référer à chacun d'eux lors de situations stressantes, de réguler ou non ses émotions et de parvenir à résoudre les dilemmes et émotions émergeants lors des histoires présentées. L'analyse des représentations de l'enfant permet d'inférer le patron d'attachement de ce dernier. Par exemple, un enfant qui n'identifie pas les émotions ni les dilemmes négatifs et ne peut pas les résoudre semblerait s'apparenter à un enfant présentant un patron d'attachement insécurisé alors qu'un enfant ayant un patron sécurisé serait en mesure de le faire et de se référer à ses figures d'attachement pour y parvenir. De même, l'enfant qui perçoit son parent comme soutenant serait plus en mesure de réguler ses émotions, ce qui correspondrait au profil d'un enfant qui se sent sécure dans sa relation avec son parent pour s'y référer sans ambivalence ou crainte. Dans son étude, Splaun (2012) rapporte également qu'un enfant présentant un patron désorganisé démontrerait un score plus élevé à l'échelle de dérégulation émotionnelle.

En résumé, l'AFCS permet de décrire les représentations d'attachement de l'enfant en identifiant si le parent est soutenant ou rejetant, s'il est utilisé comme base de sécurité, s'il parvient à accepter et réguler les émotions négatives suscités par les histoires présentées et s'il se montre capable de résoudre les dilemmes et émotions qui y émergent. L'AFCS possède une fiabilité interjuge de 0,84 et un accord exact sur au moins $80 \%$ de l'ensemble des codes constituant l'outil (Splaun, Reiner, Steele, \& Murphy, 2010). La corrélation positive entre l'AFCS et le MacArthur Narrative Coding Scale (MNCS; Robinson et al., 2007), un autre système de cotation des représentations d'attachement, confirme la validité convergente de l'instrument (Splaun, 2012; Splaun et al., 2010). Les réponses des enfants ont été enregistrées sur vidéo et retranscrites. Les récits d'attachement ont été codées par deux évaluateurs accrédités de façon indépendante. L'accord entre les deux évaluateurs pour $25 \%$ de l'échantillon de l'étude plus large, mesuré à l'aide de coefficients de corrélation intra-classe $(\mathrm{CCl})$, varie entre 0,92 et 1,00 . Pour les trois enfants présentés dans cet article, les évaluateurs ont discuté de leurs différences et parvenus par la suite à un consensus.

Les trois enfants étudiés présentaient d'importantes difficultés au plan comportemental. Afin de préciser la nature des problèmes de comportement de ces enfants, la Liste de vérification du 
comportement des jeunes de 6 à 18 ans (Child Behavior Check-list, CBCL; Achenbach \& Rescorla, 2001) a été complétée par leurs mères. Cet instrument est composé de 113 items décrivant les difficultés émotionnelles (comportements intériorisés : anxiété-dépression, retrait-dépression, plaintes somatiques) et comportementales (comportements extériorisés : conduites agressives, briser les règles), ainsi que d'autres difficultés (problèmes de la pensée, problèmes sociaux, difficultés d'attention) pouvant être actuellement présentes chez l'enfant ou dans les six derniers mois. Chaque item a été évalué selon une échelle Likert en 3 points ( 0 : ne s'applique pas; 1 : plus ou moins ou parfois vrai; 2 : toujours ou souvent vrai). Cet outil, fréquemment employé en recherche, utilise un modèle à huit facteurs et démontre une excellente cohérence interne pour l'ensemble des échelles (Heubeck, 2000). L'instrument possède une excellente fidélité interjuge (0,96 pour les items concernant les troubles spécifiques; $p<0,001)$. De même, la fidélité test-retest à une semaine d'intervalle s'étend entre 0,95 et 1,00 pour les items liés à des problèmes spécifiques (Achenbach \& Rescorla, 2001). Une étude effectuée par Moss et al. (2009) a permis d'établir une association entre les représentations d'attachement, telles qu'évaluées par une méthode narrative, les problèmes de comportement et la désorganisation de l'attachement chez les enfants d'âge scolaire. Dans leur recherche, les représentations caractérisées par des thèmes conflictuels prédisaient les comportements extériorisés et les problèmes de comportement en général. Bien que les outils utilisés pour décrire les représentations d'attachement et les comportements diffèrent de ceux de la présente analyse de cas, les résultats permettent d'alimenter la réflexion concernant les trois enfants présentés. Splaun (2012) rapporte une association entre l'échelle de dérégulation émotionnelle de l'AFCS et l'échelle de comportements externalisés du CBCL complété par les mères, laissant ainsi envisager que les enfants présentant un score élevé à l'échelle de dérégulation émotionnelle de l'AFCS ont tendance à présenter des difficultés comportementales dans leur vie.

Enfin, une étude du dossier pédopsychiatrique des trois enfants a permis également de documenter les diagnostics comorbides au TRA. Les rapports psychiatriques et psychologiques ont été consultés. Une recension de leurs diagnostics selon la quatrième édition révisée du Manuel diagnostique et statistiques des troubles mentaux (DSM-IV-TR; APA, 2000) a été effectuée dans le but d'étayer les données concernant leur fonctionnement (voir Tableau 1). Les prénoms des enfants ont été modifiés afin de préserver leur anonymat. 
Insérer ici Tableau 1

PRÉSENTION ET ANALYSE DE CAS CLINIQUES

Julie

Julie est une fillette de 9 ans et 5 mois qui présente des comportements agressifs depuis plusieurs années. Elle bénéficie de services en pédopsychiatrie et a déjà été hospitalisée à cause d'importantes désorganisations comportementales. Julie a été exposée à de la violence familiale avant la séparation de ses parents qui a eu lieu lorsqu'elle avait deux ans. Par la suite, elle vit avec sa mère chez les parents de celle-ci et voit son père en garde partagée jusqu'à l'âge de sept ans. À cet âge, le père se voit enlever la garde de l'enfant par les services de protection de la jeunesse pour maltraitance. Les contacts avec le père deviennent alors très limités, celui-ci ayant été agressif avec certains intervenants et refusant les visites supervisées. La mère n'a pas été en mesure de protéger l'enfant des comportements violents du père durant les sept premières années de sa vie lors desquelles Julie a également subi de nombreux déménagements et procédures juridiques concernant la garde. Durant cette période, l'enfant débute sa scolarité et des problèmes importants de comportements sont rapportés. Elle vivra de multiples suspensions pour agressivité qui mènent à son hospitalisation en pédopsychiatrie. À l'âge de neuf ans, Julie grogne pour se faire comprendre, se désorganise à la moindre contrariété ou frustration, présente un retard d'apprentissage et des difficultés d'attention, démontre un grand désir de plaire, des comportements sexualisés en présence de garçons de son âge ainsi qu'une importante anxiété. La fillette a un faible contrôle des impulsions, a tendance à être contrôlante avec les adultes et a de la difficulté à respecter l'autorité. Elle n'aurait pas d'amis de son âge. Julie reçoit des services en pédopsychiatrie et bénéficie d'un suivi (psychothérapie individuelle et traitement pharmacologique) ainsi que du soutien de la protection de la jeunesse (support en travail social). Elle poursuit sa scolarité en classe spécialisée pour enfants ayant un trouble d'attachement (classe fréquentée par un nombre réduit d'enfants avec soutient en orthopédagogie et en éducation spécialisée).

Les résultats de Julie au CBCL complété par la mère confirment la présence de problèmes importants (voir Tableau 2). De façon générale, les difficultés de l'enfant sont significatives par rapport à la moyenne des fillettes de son âge. Plus précisément, Julie présente des comportements agressifs et 
d'opposition ainsi que des difficultés attentionnelles. Les résultats aux autres échelles du CBCL sont dans la zone limite à l'exception de l'échelle de dépression.

Insérer ici Tableau 2

Concernant les représentations d'attachement, l'analyse des histoires de Julie (voir Tableaux 3 et 4) indique une perception des parents comme étant peu présents, peu soutenants et peu sollicités pour offrir du réconfort ou de l'aide lors de différents dilemmes. Plus particulièrement, le père est perçu comme rejetant et étant sujet à des réactions agressives verbales et physiques. L'évitement des comportements d'attachement et de la communication en rapport avec les deux parents n'est cependant pas significatif. En outre, la dérégulation émotionnelle est manifeste avec un score élevé concernant l'évitement des sentiments négatifs et des thèmes émergeant des histoires. Ainsi, la fillette est généralement incapable de résoudre les émotions et les thèmes négatifs. Julie peut même exprimer des contenus agressifs et bizarres dans ces histoires. Le profil de Julie évoque les résultats de l'étude de Splaun (2012) rapportant que les enfants présentant un patron désorganisé démontreraient un score plus élevé à l'échelle de dérégulation émotionnelle. II appuie également les résultats de la recherche de Moss et al. (2009) indiquant que la présence de thèmes conflictuels dans les histoires des enfants, étant plus fréquemment observée chez les enfants ayant un patron d'attachement désorganisé, prédit les manifestations de comportements externalisés et de problèmes comportementaux de façon générale.

Le profil de Julie est donc caractérisé par la présence de comportements externalisés cliniquement significatifs (comportements agressifs), des problèmes attentionnels et, dans une moindre mesure, de troubles internalisés (anxiété et plaines somatiques). Ses représentations d'attachement évoquent des parents peu soutenants, avec l'image d'un père pouvant être rejetant et agressif. Les parents ne sont pas sollicités lors de dilemme comme source de réconfort. Julie ne parvient pas à réguler les émotions suscitées par les histoires laissant même émerger des contenus agressifs et bizarres. Elle a tendance à éviter les émotions et thèmes négatifs et ne parvient pas à les résoudre de manière satisfaisante. Ce profil laisse envisager des représentations parentales peu sécurisantes et semble s'approcher d'un possible attachement insécurisé, voire désorganisé.

-Insérer ici Tableaux 3 et 4 
Pierre est un garçon âgé de 10 ans et 10 mois. II vit avec sa mère biologique et ses deux sœurs aînées. Son père biologique réside dans une île des Caraïbes et est absent de sa vie depuis l'âge de deux ans. La mère de Pierre a vécu avec sa propre mère jusqu'au décès de celle-ci. Auparavant, elle a quitté le Québec pour suivre sa mère à l'étranger durant sept ans. C'est à cette période qu'elle vit une relation tumultueuse avec un homme originaire du même pays. La dernière rupture se produit durant la grossesse de Pierre. Au moment de l'évaluation, madame reçoit de l'assistance sociale. Elle est diabétique, présente une symptomatologie dépressive et agoraphobe, et un deuil non-résolu relatif au décès de sa propre mère. Celle-ci est décédée au domicile, d'un arrêt cardiaque, lorsque Pierre était âgé de sept ans. La famille avait alors déménagé au Québec suite aux problèmes de santé de la grand-mère. Pierre a assisté au décès de sa grand-mère et aux manœuvres des ambulanciers pour tenter de la réanimer sans succès. II démontrera du mutisme les trois jours suivants. Dès sa naissance, l'enfant vit une relation très proche avec sa mère et dormira dans sa chambre jusqu'à l'âge de quatre ans. II rejoint d'ailleurs fréquemment sa mère la nuit. Des difficultés de sommeil et de l'énurésie sont présentes. Pierre n'a pas fréquenté de garderie. II demeure avec sa mère et ne sort de chez lui que pour aller à l'école. Dès son plus jeune âge, l'enfant présente de la difficulté à se séparer de sa mère ainsi qu'une crainte de s'éloigner de la maison. Paradoxalement, Pierre s'enfuit lorsqu'il est hors du domicile en compagnie de sa mère. Selon cette dernière, Pierre est parfois "dans son monde ». À l'école, des difficultés de comportements sont présentes (p. ex., fuites, comportements dangereux, s'enfermer dans un congélateur, s'infliger des blessures). L'enfant manifeste également des comportements compulsifs et des rituels, des difficultés d'attention, de l'impulsivité et une faible tolérance à la frustration. II a aussi tendance à vouloir contrôler les adultes, soit qu'il leur manifeste un besoin de proximité physique soit qu'il s'enfuit pour qu'ils le cherchent. Une surveillance constante est donc nécessaire pour cet enfant. De plus, Pierre s'oppose régulièrement aux consignes des adultes et fait des crises lorsque frustré. C'est un enfant solitaire qui n'a pas créé de liens d'amitié avec d'autres enfants. Pierre a été hospitalisé en pédopsychiatrie et bénéficie d'un suivi (psychothérapie individuelle et traitement pharmacologique). La mère est également en attente de services en psychiatrie. Pierre fréquente une classe régulière, mais son orientation en classe spécialisée est prévue. II présente certains diagnostics comorbides (voir Tableau 1). 
Selon le questionnaire complété par la mère, Pierre manifeste des problèmes de comportements significatifs (voir Tableau 2). Au niveau des symptômes internalisés, l'anxiété et les éléments dépressifs sont cliniquement significatifs. L'enfant présente également des comportements agressifs et des problèmes de la pensée. Les difficultés attentionnelles de l'enfant se trouvent dans la zone limite. En ce qui a trait aux représentations d'attachement de Pierre (voir Tableau 3 et 4), les parents sont perçus comme étant peu soutenants, mais non-rejetants; ils sont peu sollicités par l'enfant pour offrir du réconfort ou du support. L'évitement des comportements d'attachement et de la communication n'est pas significatif envers les deux parents. Pierre ne démontre pas non plus de difficulté de régulation émotionnelle. Les sentiments négatifs et les dilemmes ne sont pas constamment évités. En outre, la capacité de résolution de problèmes demeure très limitée. La difficulté de Pierre à se référer à l'un de ses parents face à un dilemme ne lui permet pas de parvenir à une résolution des émotions ou thèmes négatifs. Bien qu'il semble avoir une bonne capacité de régulation émotionnelle lors des récits d'attachement, son histoire de vie ainsi que les résultats au $\mathrm{CBCL}$ indiquent des problèmes importants quant aux comportements internalisés et externalisés. Pierre a également de la difficulté à se séparer de sa mère depuis son jeune âge, ce qui suggère que l'enfant a réussi à développer un lien d'attachement avec elle, même si celui-ci est caractérisé par de l'anxiété et de l'insécurité.

Le profil de Pierre est donc défini par d'importants troubles du comportement internalisés (anxiété, symptômes dépressifs), de la pensés et de comportements externalisés (agressivité). Les représentations des figures parentales sont peu soutenantes et il n'est actuellement pas en mesure de réguler les émotions et résoudre les dilemmes présentés. Ce profil permet d'inférer la présence d'un patron d'attachement insécure chez cet enfant.

Louis

Louis est un garçon âgé de 11 ans et 9 mois. II reçoit des services en pédopsychiatrie depuis son hospitalisation à l'âge de 9 ans suite à une expulsion du milieu scolaire pour des problèmes d'agressivité. Il fut de nouveau hospitalisé à l'âge de dix ans, une détérioration au plan psychologique (pensées et gestes suicidaires) étant présente malgré les interventions dispensées. L'intervention de la protection de la jeunesse fut alors demandée. Louis vit avec ses deux parents biologiques, sa sœur et son frère cadet. La mère se décrit comme une artiste ayant cessé de travailler à la naissance des enfants. Elle présente 
de l'anxiété, des difficultés d'attention et d'organisation, de l'impulsivité et des éléments dépressifs. Elle exprime être dépassée par les enfants et avoir encore de la difficulté à accepter le décès de ses deux sœurs (à l'âge de 6 et 20 ans d'une maladie génétique). Le père se déplace souvent dû à son travail. Celui-ci se décrit comme étant anxieux, impulsif, colérique, avec une tendance à consommer de l'alcool lorsqu'il est à la maison et étant performant dans son travail. II présente des difficultés d'attention et de l'impulsivité, et a reçu une médication psychostimulante durant l'enfance. Les parents rapportent une vie familiale chaotique. Les conflits parentaux sont fréquents avec violences verbales et physiques en présence des enfants. Depuis la naissance de sa sœur cadette (enfant décrite par les parents comme étant vive d'esprit, sûre d'elle et active) lorsqu'il avait un an, Louis, enfant plutôt effacé, semble ne pas avoir réussi à prendre sa place. Les conflits avec sa sœur seraient courants. Selon les parents, Louis deviendrait souvent agressif envers sa sœur. Le jeune frère, âgé de 3 ans, est décrit comme étant une " petite tornade », accaparant les adultes et faisant des crises fréquentes. Dès l'entrée scolaire, les difficultés de Louis se sont installées progressivement. II a même vécu de l'intimidation durant ses premières années. Des troubles du comportement ont alors émergé. Le milieu scolaire décrit un enfant qui dérange constamment en classe, est agressif physiquement et verbalement envers les pairs et les adultes, et est souvent exclut par ses amis. Des verbalisations suicidaires sont également présentes. Louis manifeste aussi des difficultés d'attention. L'enfant est impulsif, tolère mal les frustrations, peut se blesser lui-même et commettre des vols. II présente plusieurs diagnostics comorbides (voir Tableau 1). Louis est suivi en pédopsychiatrie (psychothérapie individuelle et traitement pharmacologique) et fréquente depuis plusieurs années une classe spéciale (classe fréquentée par un nombre réduit d'enfants) pour enfants ayant des troubles de santé mentale.

Selon le questionnaire complété par la mère, Louis présente des troubles internalisés avec d'importants symptômes d'anxiété et de dépression. Les comportements externalisés sont également cliniquement significatifs; il enfreint les règles et manifeste des comportements agressifs de façon récurrente (voir Tableau 2).

Les histoires à compléter permettent de constater que Louis perçoit ses figures parentales comme étant non-soutenantes et peu rejetantes; elles ne sont pas sollicitées lors des dilemmes. II se représente son père comme étant agressif verbalement et physiquement, et occasionnellement rejetant. L'évitement 
des comportements d'attachement et de la communication envers la mère est très significatif alors qu'envers le père, il demeure dans la norme. La dérégulation émotionnelle est faible et les émotions négatives ainsi que les dilemmes présentés ne sont pas évités. Louis démontre une bonne capacité à résoudre ceux-ci dans ces récits. Ici également, on peut relever le faible score obtenu à l'échelle de dérégulation émotionnelle. Les parents sont perçus comme étant non soutenants et l'évitement des comportements d'attachement et de la communication est manifeste envers la mère, figure la plus présente dans l'environnement de l'enfant depuis sa naissance (le père étant souvent absent). Toutefois, I'histoire de vie de Louis ainsi que les résultats au CBCL démontrent qu'il présente d'importantes difficultés au niveau de la régulation émotionnelle. L’AFCS révèle que Louis est en mesure de réguler les émotions et thèmes négatifs induits par les histoires. Bien que les représentations d'attachement de l'enfant paraissent peu sécurisantes, il fait preuve de bonnes capacités de régulation et de résolution de problèmes lorsqu'il élabore une histoire fictive. II importe de mentionner que Louis est le plus âgé des enfants de l'étude et a participé à des programmes d'intervention spécifiques concernant ses difficultés comportementales. En ce sens, il se peut que l'outil soit moins sensible pour son groupe d'âge ou que l'on observe l'impact des interventions reçues.

Le profil de Louis est donc caractérisé par d'importants troubles du comportement internalisés et externalisés. Les figures parentales sont perçues comme étant peu soutenantes avec évitement des comportements d'attachement et de la communication avec la figure maternelle. Cependant, il semble avoir des bonnes capacités de régulation et de résolution d'émotions négatives.

DISCUSSION ET CONCEPTUALISATION DES CAS CLINIQUES À LA LUMIÈRE DES RECHERCHES

\section{EN NEUROSCIENCES}

De façon générale, les données suggèrent la présence de plusieurs similitudes entre les trois enfants. De l'anxiété et des éléments dépressifs sont présents dans les trois cas étudiés. Des difficultés de régulation des émotions se retrouvent également chez deux sur trois d'entre eux dans les histoires à compléter. Louis, l'enfant démontrant une meilleure régulation émotionnelle au AFCS, bénéficie d'interventions spécifiques concernant l'attachement et la régulation des affects depuis au moins deux ans, autant en clinique de pédopsychiatrie qu'en milieu scolaire. II se peut qu'une amélioration des capacités de l'enfant au niveau de la régulation émotionnelle se reflète dans les récits qu'il élabore lors de 
l'administration des histoires d'attachement à compléter (p. ex., reconnaissance, expression et résolution des émotions négatives). Pourtant, les représentations des parents comme étant peu soutenants et l'évitement des comportements d'attachement et de la communication avec la mère demeurent présents. Dans le cas de Julie, la mise en place d'interventions spécifiques au niveau scolaire et pédopsychiatrique, ainsi que la sécurisation du milieu familial (séparation parentale et suspension des visites du parent agressif) semblent avoir réduit l'expression des éléments anxieux et dépressifs. Cependant, les trois enfants étudiés présentent ou ont présenté des troubles internalisés, soit de l'anxiété et des éléments dépressifs, ainsi que des difficultés de régulation émotionnelle. Ces données trouvent écho dans des recherches récentes qui font état d'impacts non-négligeables de la perturbation des relations d'attachement sur le développement et le fonctionnement de structures impliquées dans la régulation émotionnelle telle que l'amygdale de l'axe HPA (Neuville et al., sous presse).

Les trois enfants étudiés présentent également des troubles de comportement externalisés et plus précisément, des conduites agressives. En effet, les études suggèrent que les conditions adverses durant les premières années de vie donnent lieu à des relations d'attachement troublées et à une perturbation des mécanismes de régulation neuroendocrinienne (p. ex., régulation de la cortisone) (Dozier et al., 2006; Hunter, Minnis, \& Wilson, 2011; McBurnett, Lahey, Rathouz, \& Loeber, 2000). Cette perturbation de la régulation neuroendocrinienne altère en retour le fonctionnement des structures impliquées dans la régulation émotionnelle (axe HPA) ainsi que dans de nombreux processus cognitifs (p. ex., l'hippocampe, structure impliquée dans la mémorisation, le contrôle attentionnel et les habiletés visuo-spatiales).

Concernant les apprentissages, les trois enfants ont des retards académiques ainsi qu'un diagnostic de trouble déficitaire de l'attention avec hyperactivité-impulsivité (TDAH). Selon certaines études (Damen, Herpetz-Dahlmann, \& Konrad, 2012; Kreppner, O'Connor, \& Rutter, 2001), les enfants présentant un TRA démontrent des symptômes semblables à ceux du TDAH. Ce sont les connexions profondes des lobes frontaux, responsables des fonctions exécutives (contrôle attentionnel, contrôle des impulsions, anticipation et planification, mémoire de travail) et les structures du système limbique (axe HPA) qui seraient en cause plutôt qu'un dysfonctionnement au niveau frontal. En effet, ces études suggèrent une dysfonction limbique primaire au TDAH chez les enfants présentant un TRA contrairement aux autres enfants ayant un TDAH sans TRA (Damen et al., 2012; Kreppner et al., 2001). En outre, la perturbation de 
la régulation des mécanismes de sécrétion de cortisol a des effets délétères sur le développement et le fonctionnement de l'hippocampe pouvant aller jusqu'à induire son atrophie. Ces altérations ont alors d'importantes répercussions sur les capacités de mémorisation et d'apprentissage de l'individu (Perry, 2001; Polan \& Hoffer, 2008).

Au niveau des habiletés sociales, les mères participant à l'étude n'ont pas rapporté de difficultés significatives au questionnaire administré. Toutefois, on note dans l'histoire développementale, des difficultés aux plans des relations amicales et des compétences sociales (p. ex., absence d'amis à l'école, conflits avec les pairs). De plus, deux des participants fréquentent une classe à effectif réduit au moment de l'étude et bénéficient de soutien aux habiletés sociales et d'un encadrement rapproché pour limiter les possibilités de conflits avec les pairs. Pierre présente de l'anxiété de séparation et refuse de s'éloigner de la maison. II est également décrit comme étant un enfant solitaire ayant des difficultés de socialisation. II bénéficie d'heures d'accompagnement individuel en éducation spécialisée en classe. Certains symptômes suggèrent donc la présence de difficultés au plan social chez cet enfant bien que celles-ci ne soient pas rapportées par la mère. Plusieurs études soulignent d'ailleurs la présence d'une association entre la qualité de l'attachement et la qualité et la quantité des relations avec les pairs chez les enfants d'âge scolaire (Berlin \& Cassidy, 1999; Berlin, Cassidy, \& Appleyard, 2008; Bureau \& Moss, 2010; Elicker, Englund, \& Sroufe, 1992; Lewis \& Feiring, 1989; Thompson, 2008). Des altérations au niveau du cortex frontal sont également décrites chez les enfants présentant des difficultés à exprimer et développer de la sympathie, de l'embarras et de la culpabilité, ainsi qu'à accéder à une compréhension des règles sociales (Anderson et al., 1999). Les difficultés rapportées chez les trois enfants concernant l'attention, le contrôle des impulsions et les habiletés sociales convergent vers des perturbations du fonctionnement des zones frontales tel qu'avancé par les recherches dans le domaine (Berlin \& Cassidy, 1999; Berlin et al., 2008; Bureau \& Moss, 2010; Damen et al., 2012; Elicker et al., 1992; Kreppner et al., 2001; Lewis \& Feiring, 1989; Thompson, 2008). Ainsi, l'ajout de structures encadrantes et l'adaptation des milieux (p. ex., classe fréquentée par un nombre réduit d'enfants, accompagnement individualisé) semblent être des mesures nécessaires et contribuer à soutenir la qualité des relations avec les pairs.

L'analyse des histoires à compléter permettant d'accéder aux représentations d'attachement indiquent certaines particularités. En effet, dans les trois cas, les parents (mère et père biologiques) sont 
perçus comme peu soutenants émotionnellement lors de situations conflictuelles. Ils peuvent également être perçus comme rejetants et, pour deux des pères, agressifs physiquement et verbalement. Pour l'ensemble des enfants, la recherche de proximité auprès des figures principales d'attachement en cas de conflit (mère et père biologiques) est absente ou peu significative. Les parents ne sont donc pas perçus par ces enfants comme étant une base de sécurité. Pour leur part, les enfants ne semblent pas capables de se servir de leurs parents comme source de réconfort lorsque le système d'attachement est activé par une situation stressante. Les représentations des enfants étudiées ressemblent donc à des représentations d'attachement insécurisées. L'un des cas étudié, Julie, présente un profil avec dérégulation émotionnelle, évitement important des dilemmes et des émotions négatives avec présence de thèmes bizarres et conflictuels. La fillette a une faible capacité à résoudre les conflits. Un tel profil pourrait s'apparenter à celui d'enfants présentant une désorganisation au niveau de l'attachement. II existerait en effet une association directe entre les représentations d'attachement caractérisées par des thèmes conflictuels exprimées lors des narratifs d'attachement, les problèmes de comportement ainsi que la désorganisation de l'attachement chez l'enfant d'âge scolaire (Moss et al., 2009). De même, un score élevé à l'échelle de dérégulation émotionnelle de l'AFCS serait associé à un attachement désorganisé (Splaun, 2012). L'analyse des récits d'attachement suggère la présence des représentations de type insécurisé pour les trois enfants étudiés. Cependant, comme le suggère Zeanah et al. (2005), l'évaluation du degré de formation du lien d'attachement chez les enfants présentant un TRA permettrait de nuancer et mieux comprendre les données issues des mesures de l'attachement.

Concernant l'histoire de vie des enfants étudiés, il demeure difficile de déterminer si les conditions correspondent à celles décrites dans les critères diagnostic du TRA (APA, 2013). Le trouble d'attachement peut survenir lorsque l'enfant ne peut développer un lien significatif avec une personne disponible et sensible, de par l'absence ou le changement répété de figure de soins en bas âge dans trois contextes, soit lors de la fréquentation de certaines institutions comme les orphelinats, lors de changements répétés de la figure de soins principale ( $p$. ex., plusieurs familles d'accueil) ou lorsque l'enfant habite avec des soignants identifiables, mais extrêmement négligents. Les conditions de vie des trois cas étudiés s'apparentent effectivement à des conditions adverses, peu sécurisantes, avec présence de maltraitance et de traumas. Toutefois, ces conditions pourraient être insuffisantes pour un tel 
diagnostic et être associées à un attachement formé, même si insécurisé ou désorganisé, plutôt qu'à un TRA (Hanson \& Spratt, 2000). Tel que relevé par Lyons-Ruth (2015), il est possible de se questionner sur la définition de la négligence extrême en termes d'attachement: Est-ce de la négligence physique et émotionnelle ou l'on fait référence à un manque d'interaction avec les figures de soins? Comment peut-on évaluer cette donnée de façon précise dans les trois cas étudiés? L'absence d'outils standardisés et fiables permettant d'identifier les enfants qui présentent un TRA, les étiologies de certains comportements sociaux atypiques difficiles à discriminer les uns des autres (p. ex. trouble de régulation des processus neurosensoriels, TDAH sévère, TSA, anxiété sévère, trouble sévère du langage) et le fait que les diagnostics comorbides au TRA sont fréquents, complexifient ce diagnostic (Zephyr, Monette, Cyr, \& StAndré, 2015). D'ailleurs, pour certains auteurs, le TRA ressemble davantage aux comportements des enfants ayant formé un attachement désorganisé et pourrait être considéré comme une indication de désorganisation extrême de par l'intensité des comportements observés (van IJzendoorn \& BakermansKranenburg, 2003). L'analyse des cas présentée ne suggère l'éventualité d'une désorganisation de l'attachement que pour un des enfants étudiés. Néanmoins, les profils de ces trois enfants confirment que le TRA demeure encore un diagnostic complexe et méconnu.

\section{LIMITES ET PISTES DE RECHERCHE FUTURES}

Concernant les outils choisis, bien que le CBCL soit fréquemment utilisé en recherche, cet inventaire présente certaines limites. En effet, le questionnaire a été rempli par les mères des enfants participant à l'étude, elles-mêmes impliquées dans la relation d'attachement avec leurs enfants. Bien qu'elles complètent l'outil au meilleur de leurs connaissances, les items rapportés demeurent subjectifs et peuvent être influencés par leur désir de bien paraître socialement. Par ailleurs, lorsque les enfants étudiés fréquentent un milieu adapté (p. ex., classe fréquentée par un nombre réduit d'enfants; accompagnement individualisé), les comportements problématiques sont généralement diminués ou atténués, et donc ne ressortent pas au moment où le questionnaire est complété. De même, si l'enfant a bénéficié d'interventions spécialisées avant l'étude, il se peut que des améliorations soient présentes et que les difficultés manifestées auparavant n'apparaissent plus à l'inventaire.

Dans le même ordre d'idée, le système de cotation des récits d'attachement employé (AFCS; Splaun, 2012; Splaun et al., 2010) est relativement peu connu et seulement quelques études l'ont utilisé. 
Cependant, I'AFCS demeure un outil cliniquement intéressant qui devrait être supporté par des recherches plus exhaustives. En effet, l'analyse des représentations d'attachement des trois cas étudiés permet d'explorer les perceptions que ces enfants peuvent avoir de chacun de leurs parents ainsi que leur façon de transiger avec les dilemmes activant leur système d'attachement. En revanche, l'AFCS ne permet pas de faire un rapprochement direct entre ces représentations et les patrons d'attachement provenant de la psychologie développementale.

Une limite importante de l'étude provient du diagnostic même de TRA des enfants étudiés. En effet, il est difficile de définir de façon précise les conditions de vie adverses, de négligence extrême et de maltraitance pouvant amener à ce diagnostic (Lyons-Ruth, 2015). En ce sens, les histoires de vie chaotiques avec des milieux familiaux dysfonctionnels pourraient être des conditions insuffisantes pour expliquer un trouble d'attachement (Hanson \& Spratt, 2000). En effet, le manque d'outils standardisés pour évaluer le TRA contribue à la complexité d'un tel diagnostic (Zephyr et al., 2015).

Par ailleurs, la validité de l'étude des dossiers psychiatriques est questionnable, même s'ils constituent une source très riche d'information concernant l'histoire de vie et le fonctionnement psychologique des enfants. Les informations qu'y se trouve ne sont pas uniformes quant aux sources et aux outils d'évaluation du TRA.

Dans un autre ordre d'idées, la documentation scientifique suggère une perturbation de la régulation neuroendocrinienne chez les enfants portant un diagnostic de TRA. II serait intéressant d'ajouter aux protocoles de recherche concernant ce trouble une mesure physiologique permettant d'évaluer cette variable. Ceci permettrait d'étudier davantage les liens entre les perturbations des relations d'attachement et les altérations du fonctionnement neurologique chez les enfants ayant reçu ce diagnostic.

\section{CONCLUSION}

L'objectif de cette présentation de cas était d'illustrer que les manifestations cliniques d'enfants ayant reçu un diagnostic de TRA pouvaient suggérer d'éventuelles perturbations dans le développement ou le fonctionnement de structures cérébrales tel qu'avancé par les recherches dans le domaine des

neurosciences. Les connaissances actuelles prédisent un impact des relations d'attachement perturbées sur le développement cérébral et la santé mentale de l'individu pouvant altérer les structures cognitives supérieures (cortex frontal), le système limbique (axe HPA) et la mémoire (hippocampe). Pour ce faire, 
l'histoire développementale, les symptômes, les difficultés émotionnelles et comportementales, les représentations d'attachement et les diagnostics comorbides de trois enfants présentant un TRA ont été décrits et analysés. Chez les trois enfants, des perturbations de la régulation des comportements, des émotions et de l'attention, ainsi que des difficultés et des retards d'apprentissage sont présents. Ces manifestations suggèrent des altérations au niveau du développement et du fonctionnement de certaines structures cérébrales, structures décrites dans la recherche actuelle comme étant altérées par la présence d'un TRA chez l'individu. En effet, les symptômes au niveau de la régulation des émotions suggèrent un dysfonctionnement au niveau du système limbique et plus précisément de l'axe HPA. Ce dysfonctionnement peut avoir un impact sur l'hippocampe et le cortex frontal, ce qui pourrait affecter les capacités de mémorisation, d'apprentissage et d'attention, ainsi que les habiletés sociales de l'enfant.

L'étude des représentations d'attachement des participants a permis d'envisager la présence de représentations d'attachement insécurisées chez les trois cas analysés. La littérature mentionne la possibilité de désorganisation extrême chez les enfants présentant un TRA (van IJzendoorn \& Bakermans-Kranenburg, 2003) ou un attachement non-formé (Zeanah et al., 2005). Pour les trois cas étudiés, la possibilité d'une désorganisation n'est présente que pour un seul enfant et le degré de formation de l'attachement de ces enfants n'est pas évalué. Par ailleurs, il demeure difficile de déterminer les similitudes entre les enfants portant un diagnostic de TRA, ce qui rend ardue l'analyse de résultats et la comparaison des études (Zephyr et al., 2015). Le développement d'outils standardisés et valides permettant d'évaluer les enfants présentant un TRA est essentielle à la compréhension des difficultés de ces enfants et à la mise sur pieds d'interventions efficaces (p. ex., attachement non-formé versus attachement formé mais insécurisé).

Étant donné que les enfants ayant reçu un diagnostic de TRA risquent de présenter d'autres problèmes ultérieurement, soit à l'adolescence ou à l'âge adulte, il est important de prendre en compte l'impact que les relations d'attachement perturbées peuvent avoir sur le développement cérébral afin de mieux comprendre les atteintes émotionnelles, comportementales, scolaires et relationnelles que ces enfants peuvent présenter. L'intégration des connaissances en neurosciences avec celles de la pédopsychiatrie pourrait donner naissance à une pratique interdisciplinaire et intégrative prometteuse et 
bénéfique pour ces enfants. De futures recherches sont donc nécessaires afin de confirmer les hypothèses qui se dégagent de l'analyse des trois cas cliniques présentés. 


\section{RÉFÉRENCES}

Achenbach, T. M., \& Rescola, L. A. (2001). Manual for ASEBA School-Age Forms \& Profiles. Burlington, V T: University of Vermont, research Center for Children, Youth, \& Families.

American Psychiatry Association (2000). Diagnostic and statistical manual of mental disorders (4 ${ }^{\text {th }}$ edition revised). Arlington, VA: American Psychiatric Association Publishing.

American Psychiatry Association. (2013). Diagnostic and statistical manual of mental disorders $\left(5^{\text {th }}\right.$ edition). Washington: American Psychiatric Association.

Anderson, S. W., Bechara, A., Damasio, H., Tranel, D., \& Damasio, A. R. (1999). Impairment of social and moral behavior related to early damage in human prefrontal cortex. Nature Neuroscience, 2(11), 1032-1037.

Berlin, L. J., \& Cassidy, J. (1999). Relations among relationships: Contributions from attachment theory and research. Dans J. Cassidy \& P. R. Shaver (Éds.), Handbook of attachment: Theory, research, and clinical applications (1ère éd.; pp. 688-712). New York, NY: Guilford Press.

Berlin, L. J., Cassidy, J., \& Appleyard, K (2008). The influence of attachment security and related constructs in infancy and early childhood. Dans Cassidy J., \& Shaver P. R. (Éds.), Handbook of attachment: Theory, research, and clinical applications (2ème éd.; pp. 383-416). New York, NY: Guilford Press.

Bretherton I., \& Munholland, K. A. (2008). Internal working model in attachment relationships: Elaborating a central construct in attachment theory. Dans J. Cassidy, \& P. R. Shaver (Éds.), Handbook of attachment: Theory, research, and clinical applications, (2ème éd.; pp. 102-127). New York, NY: Guilford Press.

Bretherton, I., Ridgeway, D., \& Cassidy, J. (1990). Assessing internal working models of the attachment relationship: An Attachment Story Completion Task for 3-year-olds. Dans T. Greenberg, D. Cicchetti, \& E. M. Cummings (Éds.), Attachment in the Preschool Years: Theory, Research, and Intervention (pp. 273-308). Chicago, IL: University of Chicago Press.

Bureau, J. F., Béliveau, M. -J., Moss, E., \& Lépine, S. (2006). Association entre l'attachement mère-enfant et les récits d'attachement à la période scolaire. Revue canadienne des sciences du comportement, 38(1), 50-62. 
Bureau, J. -F., \& Moss E. (2010). Behavioral precursor of attachment representations in middle childhood and links with child social adaptation. British Journal of Developmental Psychology, 28(3), 657677.

Chugani, H. T., Behen, M. E., Muzik, O., Juhasz, C., Nagy, F., \& Chugani, D. C. (2001). Local brain functional activity following early deprivation: A study of postinstutitionalized Romanian orphans. Neurolmage, 14(6), 1290-1301.

Coleman, P. K. (2003). Reactive Attachment Disorder in the context of the family: A review and call for further research. Emotional and Behavioral Difficulties, 8(3), 205-216.

Damen, B., Pütz, V., Herpetz-Dahlmann, B., \& Konrad, K. (2012). Early pathogenic care and the development of ADHD-like symptoms. Journal of Neural Transmission, 119(9), 1023-1036.

DeKlyen, M., \& Greenberg, M. T. (2008). Attachment and psychopathology in childhood. Dans J. Cassidy \& P. R. Shaver (Éds.), Handbook of attachment: Theory, research, and clinical applications ( $2^{\text {nd }}$ ed.; pp. 637-665). New York, NY: Guilford Press.

Dozier, M., Manni, M., Gordon, M. K., Peloso, E., Gunnar, M. R., Stovall-McClough, K. C., Eldreth, D., \& Levine, S. (2006). Foster children's diurnal production of cortisol: An exploratory study. Child Maltreatment, 11(2), 189-107.

Elicker, J., Englund, M., \& Sroufe, L. A. (1992). Predicting peer competence and peer relationships in childhood from early parent-child relationships. Dans R. D. Parke \& G. W. Ladd (Éds.), Familypeer relationshilps: mode of linkage, (pp. 77-106). Hillsdale, NJ: Lawrence Erlbaum Associates.

Egeland, B., \& Carlson, E. (2004). Attachment and psychopathology. Dans L. Atkinson \& S. Goldberg (Éds.), Attachment issues in psychopathology and intervention (pp. 27-48). Mahwah, NJ: Lawrence Erlbaum Associates Publishers.

Fenoglio, K. A., Chen, Y., \& Baram, T. Z. (2006). Neuroplasticity of the hypothalamic-pituitary-adrenal axis early life requires recurrent recruitment of stress-regulating brain regions. The Journal of Neuroscience, 26(9), 2434-2442.

Gleason, M. M., \& Zeanah, C. H. (2010). Assessing infants and toddlers. Dans M. K. Dulcan (Éd.), Dulcan's textbook of child and adolescent psychiatry (pp. 3-14). Arlington, VA: American Psychiatry Publishing. 
Govindan, R. M., Behen, M. E., Helder, E., Makki, M. I., \& Chugani, H. T. (2010). Altered water diffusy in cortical association tracts in children with early deprivation identification with tract-based spatial statistics (TBSS). Cerebral Cortex, 20(2), 561-569.

Grych, J.H., Waschsmuth-Sclaefer, T., \& Klockow, L. (2002). Interparental aggression and young children's representations of family relationships. Journal of Family Psychology, 16(3), 259-272.

Hanson, R. F., \& Spratt, E. G. (2000). Reactive Attachment Disorder: What we know about the disorder and implications for the treatment. Child Maltreatment, 5(2), 137-145.

Hunter, A. L., Minnis, H., \& Wilson, P. (2011). Altered stress responses in children exposed to early adversity: A systematic review of salivary cortisol studies. The International Journal on the Biology of the Stress, 14(6), 614-626.

Klasseens, E. R. (2010). Bouncing back - Trauma and the HPA-axis in healthy adults. European Journal of Psychotraumatology, $\quad$ Supplement $1, \quad 10.3402 /$ ejpt.v1i0.5844: http://doi.org/10.3402/ejpt.v1i0.5844

Klasseens, E. R., van Noorden, M. S., Giltay, E. J., van Pelt, J., van Veen, T., \& Zitman F. G. (2009). Effects of childhood trauma on HPA-axis reactivity in women free of lifetime psychopathology. Progress in Neuro-Psychopharmacology \& Biological Psychiatry, 33, 889-894.

Kreppner, J. M, O'Connor, T. G., \& Rutter, M. (2001). Can inattention/overactivity be an institutional deprivation syndrome? Journal of Abnormal Child Psychology, 29(6), 513-528.

Heubeck, B. G. (2000). Cross-cultural generalizability of CBCL syndromes across three continents: From USA and Holland to Australia. Journal of Abnormal Child Psychology, 28(5), 439-450.

Lavie, N., \& de Fockert, J. (2006). Frontal control of attentional capture in visual capture in visual search. Visual Cognition, 14(4-8), 863-876.

Levy, B. J., \& Wagner, A. D. (2011). Cognitive control and right ventorlateral prefrontal cortex: Reflexive reorienting, motor inhibition, and action updating. Annals of The New York Academy of Sciences, $1224,40-62$.

Lewis, M., \& Feiring, C. (1989). Infant, mother, and infant-mother interaction behavior and subsequent attachment. Child Development, 60(4), 831-837. 
Ludy-Dobson, C., \& Perry B. D. (2010). The role of healthy relational interaction in buffering the impact of childhood trauma. In E. Gilwith (Ed.), Working with children to heal interpersonal trauma: The power of play (pp. 26-42). New York, NY: Guilford Press.

Lyons-Ruth, K. (2015). Commentary: Should we move away from an attachment framework for understanding disinhibited social engagement disorder (DSED)? A commentary on Zeanah and Gleason (2015). Journal of Child Psychology and Psychiatry, 56(3), 223-227.

Main, M., Kaplan, N., \& Cassidy, J. (1985). Security in infancy, childhood, and adulthood: A move to the level of representation. Monographs of the Society for Research in Child Development, 50(1-2), 66-104.

Mayseless, O. (2005). Ontogeny of attachment in middle childhood: Conceptualization of normative changes. Dans K. A. Kern \& R. A. Richardson (Éds.), Attachment in middle childhood (pp. 1-23). New York, NY: Guilford Press.

McBurnett, K., Lahey, B. B., Rathouz, P. J., \& Loeber, R. (2000). Low salivary cortisol and persistent aggression in boys referred for disruptive behavior. Archives of General Psychiatry, 57(1), 38-43.

Millward, E., Kennedy, E., Towlson, K., \& Minnis, H. (2006). Reactive Attachment Disorder in looked-after children. Emotional and Behavioral Difficulties, 11(6), 273-279.

Minnis, H., Marwick, H., Arthur, J \& McLaughlin, A. (2006) Reactive Attachment Disorder: A theoretical model beyond attachment. European Child and Adolescent Psychiatry, 15(6), 337-342.

Moss, E., Bureau, J. F., Béliveau M.J., Zdebik, M., \& Lépine, S. (2009). Links between children's attachment behavior at early school-age, their attachment-related representations, and behavior problems in middle childhood. International Journal of Behavioral Development, 33(2), 155-166.

Neuville, V., Puentes-Neuman, G, \& Terradas, M. M. (sous presse). L'impact des relations précoces sur le développement cérébral : le trouble réactionnel de l'attachement vu par les neurosciences. La psychiatrie de l'enfant.

Newman, L., \& Mares S. (2007). Recent advances in the theories and interventions with attachment disorders. Child and Adolescent Psychiatry, 20(4), 343-348.

O'Connor, T. G., \& Zeanah C. H. (2003). Current perspectives on attachment disorders: Rejoinder and synthesis. Attachment \& Human Development, 5(3), 321-326. 
Oppenheim, D. (2006). Child, parent, and parent-child emotion narratives: Implications for developmental psychopathology. Development and Psychopathology, 18, 771-790.

Perry, B. D. (2001). The neurodevelopmental impact of violence in childhood. Dans D. Schetky \& E. P. Benedek (Éds.), Textbook of child and adolescent forensic psychiatry (pp. 221-238). Arlington, VA: American Psychiatric Press.

Pinto, V., Costa, J. C., Morgado, P., Mota, C., Miranda, A., Bravo, F. V., Oliveira, T. G., Cerqueira, J. J., \& Sousa, N. (2015). Differential impact of chronic stress along the hippocampal dorsal-ventral axis. Brain Structure \& Function, 220(2), 1205-1212.

Polan, H. J., \& Hofer, M. R. (2008). Psychobiological origins of infant attachment and its role in development. Dans J. Cassidy \& P. R. Shaver (Éds.), Handbook of attachment: Theory, research, and clinical applications, (2ème éd.; pp. 383-416). New York, NY: Guilford Press.

Robinson, J., Mantz-Simmons, L., MacFie, J., Kelsay, K., Holmberg, J., \& the MacArthur Narrative Working Group. (2007). MacArthur Narrative Coding Manual. Unpublished manuscript: University of Colorado Health Sciences Center, Denver, Colorado.

Reiner, I., \& Splaun, A. K. (2008). Story Stems Attachment-Focused Coding System. Unpublished manuscript, The New School for Social Research, New York, New York.

Splaun, A. K. (2012). The brief attachment-focused coding system for story stems: A validity study (Thèse de doctorat) Repéré dans http://ezproxy.usherbrooke.ca/login?url=https://search-proquestcom.ezproxy.usherbrooke.ca/docview/1022644818?accountid=13835

Splaun, A.K., Reiner, I., Steel, M., \& Murphy, A. (2010). The congruence of parents and their children's representations of their relationship. New School Psychology Bulletin, 7(1), 51-62.

Thompson, R.A. (2008). Early attachment and later development. Familiar questions, new answers, Dans J. Cassidy \& P. R. Shaver (Éds.), Handbook of attachment: Theory, research, and clinical applications, (2ème éd.; pp. 348-365). New York, NY: Guilford Press.

Steele, M., Steel, H., Woolgar, M., Yabsley, S, Fonagy, P., Johnson, D., \& Croft, C. (2003). An attachment perspective on children's emotion narratives: links across generations. Dans R. N. Emde, D. P. Wolf, \& D. Oppenheim, D. (Éds.), Revealing the inner worlds of young children (pp. 163-181). New York, NY: Oxford University Press, 
van IJzendoorn, M. H. \& Bakermans-Kranenburg, M. J. (2003). Attachment disorders and disorganised attachment: Similar and different. Attachment \& Human Development, 5(3), 313-320.

Zeanah, C. H., Smyke, A. T., Koga, S. F., \& Carlson, E. (2005). Attachment in institutionalized and community children in Romania. Child Development, 76(5), 1015-1028.

Zephyr, L., Monette, S., Cyr, C., St-André, M. (2015). Coup d'œil sur les troubles de l'attachement. Les troubles de l'attachement durant l'enfance : état des lieux. Observatoire sur la maltraitance envers $\begin{array}{llllll}\text { les enfants. } & \text { Récupéré le } & 10 & \text { août } & 2017 & \text { de }\end{array}$ http ://observatoiremaltraitance.ca/Pages/Coup_d'oeil_sur_les_troubles_de_l'attachement_durant _\%20l'enfance. 


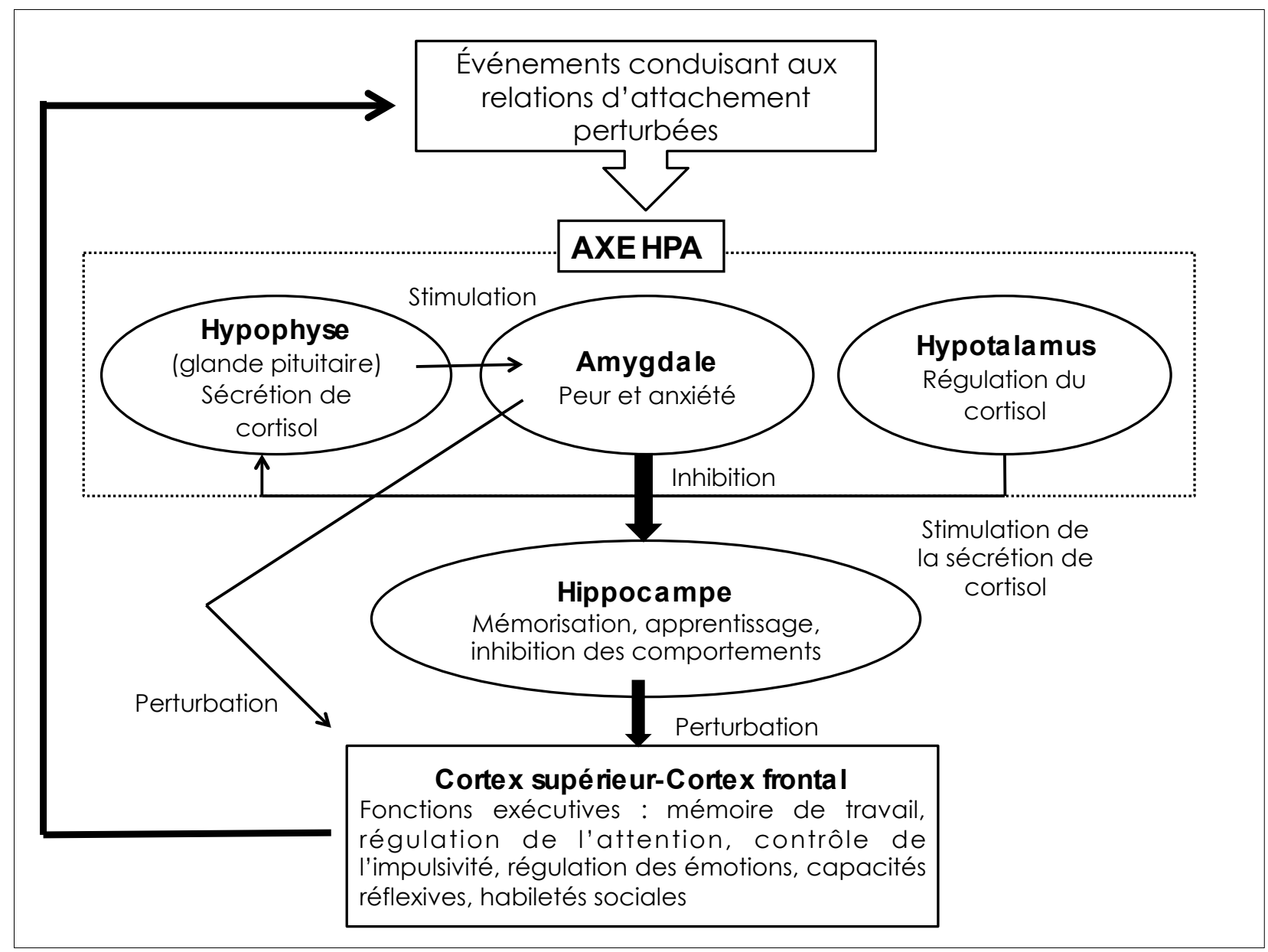

Figure 1. Modélisation de l'impact des relations d'attachement perturbées sur les structures cérébrales. 
Tableau 1

Diagnostics selon le DSM-IV-TR présentés chez les enfants en comorbidité au TRA

\begin{tabular}{|c|c|c|}
\hline Enfant & Âge & Diagnostics comorbides \\
\hline Julie & 9 ans, 5 mois & $\begin{array}{l}\text { Trouble d'adaptation avec perturbation mixte des émotions et } \\
\text { des conduites } \\
\text { Trouble déficitaire de l'attention avec hyperactivité-impulsivité } \\
\text { (TDAH) } \\
\text { Retard d'apprentissage }\end{array}$ \\
\hline Pierre & 10 ans, 10 mois & $\begin{array}{l}\text { TDAH } \\
\text { Anxiété } \\
\text { Retard d'apprentissage }\end{array}$ \\
\hline Louis & 11 ans, 9 mois & $\begin{array}{l}\text { TDAH } \\
\text { Trouble anxio-dépressif } \\
\text { Trouble oppositionnel avec provocation } \\
\text { Retard d'apprentissage }\end{array}$ \\
\hline
\end{tabular}


Tableau 2

Scores T obtenus par les enfants aux différentes échelles du CBCL

\begin{tabular}{clll}
\hline Échelle (percentile) & Julie & Pierre & Louis \\
\hline Anxiété & $66(\mathrm{~L})$ & $70(\mathrm{C})$ & $85(\mathrm{C})$ \\
Dépression & $60(\mathrm{~N})$ & $76(\mathrm{C})$ & $79(\mathrm{C})$ \\
Somatisation & $66(\mathrm{~L})$ & $53(\mathrm{~N})$ & $50(\mathrm{~N})$ \\
Problèmes sociaux & $67(\mathrm{~L})$ & $62(\mathrm{~N})$ & $60(\mathrm{~N})$ \\
Problèmes de la pensée & $66(\mathrm{~L})$ & $78(\mathrm{C})$ & $61(\mathrm{~N})$ \\
Problèmes attentionnels & $70(\mathrm{C})$ & $69(\mathrm{~L})$ & $59(\mathrm{~N})$ \\
Délinquance & $69(\mathrm{~L})$ & $60(\mathrm{~N})$ & $76(\mathrm{C})$ \\
Comportements agressifs & $79(\mathrm{C})$ & $70(\mathrm{C})$ & $78(\mathrm{C})$ \\
\hline Troubles internalisés & $67(\mathrm{~L})$ & $71(\mathrm{C})$ & $75(\mathrm{C})$ \\
Troubles externalisés & $74(\mathrm{C})$ & $69(\mathrm{~L})$ & $76(\mathrm{C})$ \\
\hline Total & $71(\mathrm{C})$ & $73(\mathrm{C})$ & $72(\mathrm{C})$ \\
\hline
\end{tabular}

Note. $(\mathrm{N})$ = Zone non clinique : Les difficultés observées chez le jeune ne sont pas plus présentes que chez les autres enfants du même groupe d'âge; $(L)=$ Zone limite : Présence de difficultés auxquelles il convient de porter attention (Score $T$ supérieur à la moyenne, soit de 64 à 69$) ;(C)$ = Zone clinique : Les difficultés observées chez le jeune sont significativement plus marquées que chez les autres enfants du même âge (Score T très supérieur à la moyenne, soit 70 et plus). 
Tableau 3

Scores moyens, minimaux et maximaux obtenus par les enfants aux Histoires d'attachement à compléter selon l'AFCS

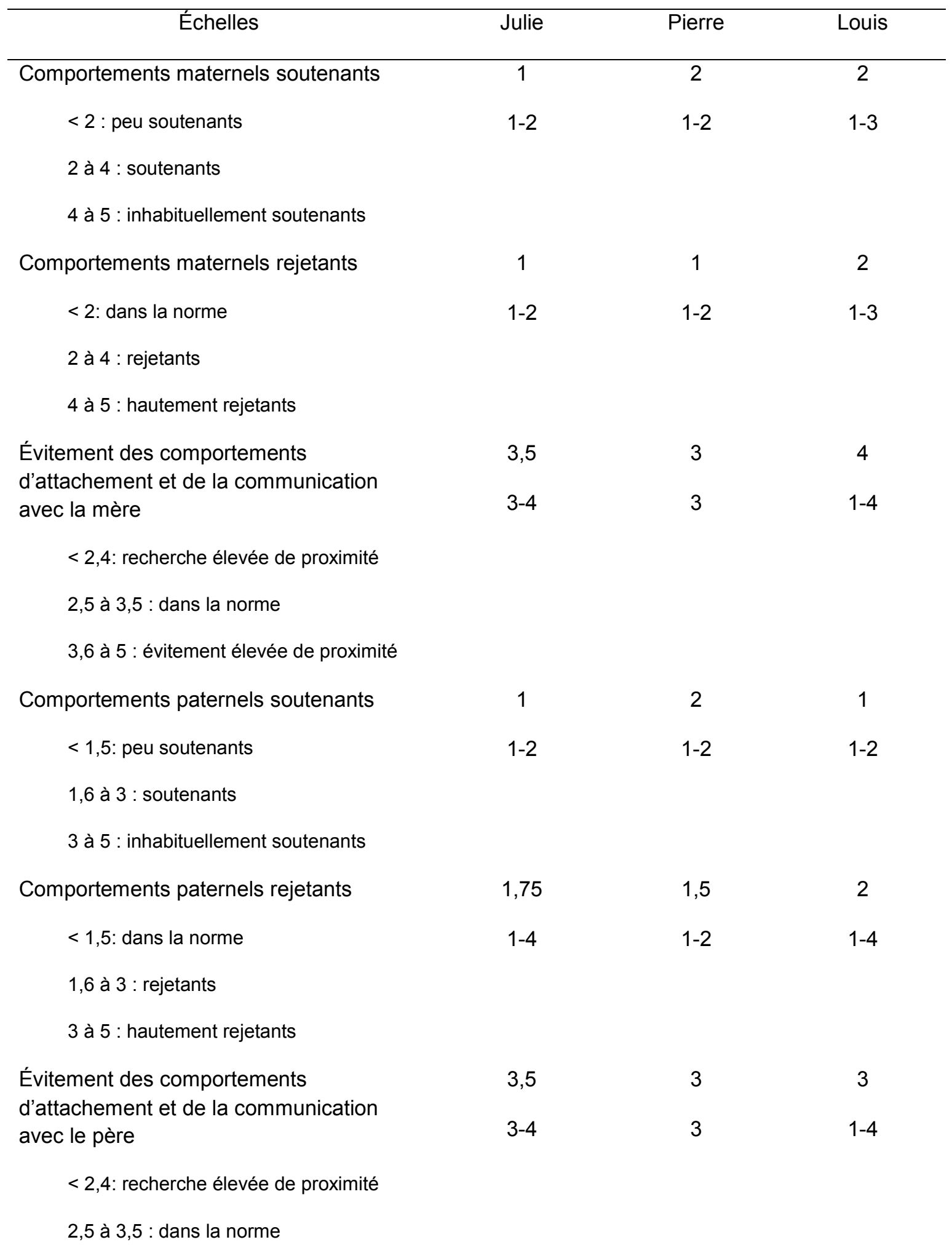


3,6 à 5 : évitement élevée de proximité

Dérégulation émotionnelle de l'enfant

$<1,8$ : faible dérégulation

1,8 à $2,5:$ quelques dérégulations

2,6 à 5 : dérégulation élevée

Évitement des sentiments négatifs et des thèmes de l'histoire

$<$ 1,9: peu d'évitement

2 à 3,5 : évitement modéré

2,6 à 5 : évitement élevé

Résolution des thèmes et émotions négatifs

$<2,1$ : absence de résolution

2,2 à 3,8 : résolution modérée

3,9 à 5 : résolution élevée
2

$1-3$

4

3-4

$1-3$

$1-2$

2

2

5

$1-3$
$2-3$

$2-5$

$\begin{array}{lll}-3 & 2-3 & 2-5\end{array}$

1 
Tableau 4

Représentations d'attachement des enfants évaluées à l'aide de l'ACFS

\begin{tabular}{|c|c|c|c|c|}
\hline Critères & Représentations d'attachement (cote AFCS) & \multicolumn{3}{|c|}{ Enfants } \\
\hline & & Julie & Pierre & Louis \\
\hline \multirow[t]{3}{*}{$\begin{array}{l}\text { Relation avec la } \\
\text { mère }\end{array}$} & $\begin{array}{l}\text { La mère n'offre aucun soutien émotionnel ou } \\
\text { concret à l'enfant ou est peu soutenante lors des } \\
\text { dilemmes présentés. }\end{array}$ & $X$ & $X$ & $\bar{X}$ \\
\hline & $\begin{array}{l}\text { La mère présente un certain rejet émotionnel ou } \\
\text { physique. Par exemple, la mère crie après } \\
\text { l'enfant ou le réprimande sur un ton un peu } \\
\text { fâché ce qui inclut la discipline, l'imposition de } \\
\text { limites adéquates et non excessives ou } \\
\text { altercation verbale intense devant l'enfant et ce, } \\
\text { même si l'enfant n'est pas directement impliqué. }\end{array}$ & & & $\mathrm{X}$ \\
\hline & $\begin{array}{l}\text { Pas d'évitement ni de recherche active de } \\
\text { proximité de la part de l'enfant ou présence d'un } \\
\text { léger évitement du comportement } \\
\text { d'attachement. }\end{array}$ & $X$ & $X$ & $X$ \\
\hline \multirow[t]{4}{*}{$\begin{array}{l}\text { Relation avec le } \\
\text { père }\end{array}$} & $\begin{array}{l}\text { Le père n'offre aucun soutien émotionnel ou } \\
\text { concret à l'enfant ou est peu soutenant lors des } \\
\text { dilemmes présentés. }\end{array}$ & $X$ & $X$ & $X$ \\
\hline & $\begin{array}{l}\text { Le père présente un certain rejet émotionnel ou } \\
\text { physique. Par exemple, il crie après l'enfant ou } \\
\text { le réprimande sur un ton un peu fâché ce qui } \\
\text { inclut la discipline, l'imposition de limites } \\
\text { adéquates et non excessives ou altercation } \\
\text { verbale intense devant l'enfant et ce, même si } \\
\text { l'enfant n'est pas directement impliqué. }\end{array}$ & $\mathrm{X}$ & $\mathrm{X}$ & $X$ \\
\hline & $\begin{array}{l}\text { Présence d'un abus verbal ou physique de la } \\
\text { part du père. }\end{array}$ & $X$ & & $X$ \\
\hline & $\begin{array}{l}\text { Pas d'évitement ni de recherche active de } \\
\text { proximité de la part de l'enfant ou présence d'un } \\
\text { léger évitement du comportement } \\
\text { d'attachement. }\end{array}$ & $\mathrm{X}$ & $\mathrm{X}$ & $\mathrm{X}$ \\
\hline \multirow[t]{3}{*}{$\begin{array}{l}\text { Habiletés à } \\
\text { reconnaître et } \\
\text { réguler les } \\
\text { émotions } \\
\text { négatives }\end{array}$} & $\begin{array}{l}\text { Dérégulation/effondrement émotionnel : l'enfant } \\
\text { démontre un contenu agressif ou bizarre, ou une } \\
\text { agression verbale extrême et/ou des formes } \\
\text { mineures d'agression physique dans les } \\
\text { histoires. }\end{array}$ & $\bar{X}$ & $\mathrm{X}$ & \\
\hline & $\begin{array}{l}\text { Évitement des thèmes et sentiments négatifs } \\
\text { avec soit expression ou évitement marqué dans } \\
\text { les histoires. }\end{array}$ & $X$ & $X$ & \\
\hline & $\begin{array}{l}\text { Résolution partielle et mineure des thèmes ou } \\
\text { des émotions négatives dans les histoires. }\end{array}$ & & & \\
\hline
\end{tabular}


Conclusion 
Le premier article de cette thèse vise à identifier les possibles perturbations neurodéveloppementales concomitantes à un TRA selon les résultats des recherches en neurosciences. Plus particulièrement, des altérations de l'activité des structures de l'axe HPA au niveau neuroendocrinien (cortisol), élément essentiel à la régulation des émotions, ainsi que des anomalies du fonctionnement des structures supérieures telles que l'hippocampe et le cortex frontal, sont relevées. Ces difficultés sur le plan de l'activité cérébrale sont reconnues pour avoir des répercussions importantes sur la performance cognitive et comportementale des enfants. En effet, les altérations du fonctionnement des structures de l'axe HPA provoquent des modifications au niveau de la libération du cortisol qui en retour, modifie l'activité d'autres structures du système limbique. Ces altérations provoquent des difficultés dans la régulation de l'anxiété et du stress, et influent également sur le bon fonctionnement des systèmes impliqués dans la mémorisation, l'attention, la régulation des comportements et les habiletés sociales (hippocampe et cortex frontal). Des altérations profondes suite à la persistance des conditions adverses sont même identifiées (p. ex., atrophie de l'hippocampe par excès de sécrétion de cortisol, Lupien et al., 1998; Perry, 2001; Polan \& Hoffer, 2008); elles pourraient devenir permanentes (Perry \& Sullivan, 2014; Thomas et al., 2001).

Les altérations neurologiques décrites par les neurosciences ont un impact au niveau émotionnel, comportemental, académique, relationnel et de la santé mentale en général, et impliquent de l'anxiété et des éléments dépressifs, des troubles du comportement, des troubles de l'attention, des troubles d'apprentissage et des difficultés dans les relations interpersonnelles. Ces difficultés se répercutant de façon importante dans toutes les sphères de fonctionnement des enfants présentant un TRA. Ceux-ci fréquentent généralement de services en pédopsychiatrie. C'est donc par l'analyse du profil clinique d'enfants ayant reçu un diagnostic de TRA recevant de 
tels services, qu'un rapprochement entre les prédicats de la recherche et la symptomatologie relevée chez ces enfants a été possible.

Le second article de cette thèse tente de mettre en évidence la présence ou l'absence de symptômes cliniques dans les sphères de la régulation des émotions, du comportement, des apprentissages et des relations interpersonnelles chez des enfants présentant un diagnostic de TRA par le biais de l'analyse de cas cliniques. Cette méthode a permis de faire une analyse approfondie des difficultés présentes chez les enfants ayant un reçu un diagnostic de TRA et de les interpréter à la lumière des atteintes possibles relevées par la recherche en neurosciences. Malgré certaines limites inhérentes à l'analyse de cas, celle-ci permet cependant de mieux illustrer le phénomène et d'offrir des suggestions pour des recherches futures.

Les comportements, les représentations d'attachement ainsi que les diagnostics portés par les enfants participants ont été analysés. Les résultats de l'analyse de cas soulignent la présence des perturbations de la régulation du comportement, des émotions, de l'attention, ainsi que des retards d'apprentissage et des difficultés interpersonnelles pour les enfants étudiés. Ces manifestations peuvent effectivement suggérer des altérations au niveau du fonctionnement de certaines structures cérébrales, structures décrites dans la recherche actuelle comme étant altérées par la présence des conditions de vie pouvant amener au diagnostic d'un TRA chez l'individu. En effet, les symptômes au plan de la régulation des émotions suggèrent un dysfonctionnement au niveau du système limbique et plus précisément de l'axe HPA, un tel dysfonctionnement pouvant se répercuter sur les capacités de mémorisation, d'apprentissage, d'attention et les habiletés sociales de l'enfant. Il peut également avoir un impact sur le fonctionnement des structures cérébrales telles que l'hippocampe et le cortex frontal. En outre, l'étude des représentations 
d'attachement des participants a permis de préciser leur façon de percevoir chacun de leur parent en terme de support et de base de sécurité ainsi que leur capacité de transiger avec les émotions et thèmes négatifs. Dans l'ensemble, les parents ne sont pas identifiés comme sécurisants ni réconfortants pour les trois cas étudiés. Même si la controverse concernant l'éventualité d'une correspondance entre le TRA et la classification des représentations d'attachement utilisée dans le domaine de la psychologie développementale persiste (Minnis et al., 2006; O’Connor, Bredenkamp \& Rutter, 1999), l'identification des représentations d'attachement de ces enfants demeure pertinente et essentielle pour orienter les interventions et mieux comprendre leur fonctionnement. Par ailleurs, la présente étude a mis en lumière les difficultés reliées au diagnostic même de TRA. Est-il question de désorganisation importante ou d'attachement nonformé? Comment rendre opérationnels des critères diagnostiques tels que «négligence extrême »? Quel outil standardisé obtient un consensus pour appuyer un tel diagnostic? De nombreuses pistes de réflexion demeurent donc à explorer.

L'ensemble des résultats de cette analyse de cas cliniques permet de proposer un rapprochement entre les résultats des recherches en neuroscience et la symptomatologie observée chez les enfants présentant un diagnostic de TRA. Cette constatation conduit à de nouvelles perspectives et génère de multiples questionnements. En effet, il devient incontournable de considérer l'impact de conditions adverses au cours des premières années de vie sur le développement cérébral des enfants pour mieux comprendre les symptômes exprimés par ceuxci. Cette perspective permet de mieux envisager les avenues d'intervention et de travailler en prévention, certaines altérations pouvant amener des troubles dans les différentes sphères de leur vie (p. ex., relationnelle, académique, émotionnelle). Développer des outils de dépistage en bas âge et des interventions visant l'établissement d'une relation d'attachement sécurisée pourrait 
également aider à prévenir des difficultés ultérieures chez de nombreux enfants. De même, il serait intéressant de questionner les services dispensés aux enfants orphelins ou placés qui, encore actuellement, subissent de nombreux déplacements, reçoivent les soins de multiples intervenants et dont les problématiques comportementales s'amplifient dans un tel contexte. Une roue sans fin peut alors s'installer sans que le développement de la sécurité d'attachement nécessaire à l'apaisement de l'enfant ne devienne l'intervention à prioriser. L'intégration des connaissances en neurosciences avec celles issues de la pratique clinique ouvre la voie à des interventions plus ciblées ainsi qu'à une pratique interdisciplinaire et intégrative très prometteuse et bénéfique pour ces enfants. De futures recherches sont donc nécessaires afin de confirmer les observations effectuées lors de l'analyse de ces trois cas cliniques. 
Références 
Achenbach, T.M., \& Rescola, L.A. (2001). Manual for ASEBA School-Age Forms \& Profiles. Burlington, V T: University of Vermont, research Center for Children, Youth, \& Families

Ainsworth, M. D., Blehar, M. C., Waters, E., \& Walls, S. (1978). Patterns of attachment: A psychological study of the strange situation. Hillsdale, NJ: Lawrence Earlbaum Associates.

American Psychiatry Association. (1980). Diagnostic and statistical manual of mental disorders ( $3^{\text {th }}$ edition revised). Arlington, VA: American Psychiatric Association Publishing.

American Psychiatry Association. (2000). Diagnostic and statistical manual of mental disorders ( $4^{\text {th }}$ ed. revised). Arlington, VA: American Psychiatric Association Publishing.

American Psychiatry Association. (2013). Diagnostic and statistical manual of mental disorders ( $5^{\text {th }}$ ed.). Arlington, VA: American Psychiatric Association Publishing.

Bowlby, J. (1969). Attachment and loss, vol.1. New York, NY: Basic Books.

Bowlby, J. (1973). Attachment and loss, vol.2: Separation. New York, NY: Basic Books.

Bowlby, J. (1980). Attachment and loss, vol.3: Loss, sadness and depression. New York, NY: Basic Books.

Borelli, J. L., David, D. H., Crowley, M. J., \& Mayes, L. C. (2010). Links between disorganized attachment classification and clinical symptoms in school-aged children. Journal of Child \& Family Studies, 19(3), 243-256.

Boris, N. W., Fueyo, M., \& Zeanah, C. H. (1997). The clinical assessment of attachment in children under five. Journal of the American Academy of Child \& Adolescent Psychiatry, 36(2), 291-293.

Boris, N. W., Zeanah, C. H., Larrrieu, J. A., Scheeringa, M. S., \& Heller, S. (1998). Attachment disorders in infancy and early childhood: A preliminary investigation of diagnostic criteria. American Journal of Psychiatry, 155(2), 295-297.

Bretherton I., \& Munholland, K. A. (2008). Internal working model in attachment relationships: Elaborating a central construct in attachment theory. In J. Cassidy \& P. R. Shaver (Eds.), Handbook of attachment: Theory, research, and clinical applications $\left(2^{\text {nd }}\right.$ ed., pp. 102127). New York, NY: Guilford Press.

Bretherton, I., Ridgeway, D., \& Cassidy, J. (1990). Assessing internal working models of the attachment relationship: An Attachment Story Completion Task for 3-year-olds. Dans T. Greenberg, D. Cicchetti, \& E. M. Cummings (Eds.), Attachment in the Preschool Years: Theory, Research, and Intervention (pp. 273-308). Chicago, IL: University of Chicago Press. 
Bureau, J. F., Béliveau, M. -J., Moss, E., \& Lépine, S. (2006). Association entre l'attachement mère-enfant et les récits d'attachement à la période scolaire. Revue canadienne des sciences du comportement, 38(1), 50-62.

Bureau, J. -F., \& Moss E. (2010). Behavioral precursor of attachment representations in middle childhood and links with child social adaptation. British Journal of Developmental Psychology, 28(3), 657-677.

Chugani, H. T., Behen, M. E., Muzik, O., Juhasz, C., Nagy, F., \& Chugani, D. C. (2001). Local brain functional activity following early deprivation: A study of postinstutitionalized Romanian orphans. NeuroImage, 14(6), 1290-1301.

Coleman, P. K. (2003). Reactive Attachment Disorder in the context of the family: A review and call for further research. Emotional and Behavioral Difficulties, 8(3), 205-216.

DeKlyen, M., \& Greenberg, M. T. (2008). Attachment and psychopathology in childhood. In J. Cassidy \& P. R. Shaver (Eds.), Handbook of attachment: Theory, research, and clinical applications ( $2^{\text {nd }}$ ed., pp. 637-665). New York, NY: Guilford Press.

Fenoglio, K. A., Chen, Y., \& Baram, T. Z. (2006). Neuroplasticity of the hypothalamic-pituitaryadrenal axis early life requires recurrent recruitment of stress-regulating brain regions. The Journal of Neuroscience, 26(9), 2434-2442.

Gleason, M. M., \& Zeanah, C. H. (2010). Assessing infants and toddlers. In M. K. Dulcan (Ed.), Dulcan's textbook of child and adolescent psychiatry (pp. 3-14). Arlington, VA: American Psychiatry Publishing.

Green, J., \& Goldwyn, R. (2002). Annotation: Attachment disorganization and psychopathology: New findings in attachment research and their potential implications for developmental psychopathology in childhood. Journal of Child Psychology and Psychiatry, 43(7), 835846.

Grych, J. H., Waschsmuth-Sclaefer, T., \& Klockow, L. (2002). Interparental aggression and young children's representations of family relationships. Journal of Family Psychology, $16(3), 259-272$.

Hanson, R. F., \& Spratt, E. G. (2000). Reactive Attachment Disorder: What we know about the disorder and implications for the treatment. Child Maltreatment, 5(2), 137-145.

Kemeny, M. E. (2003). Psychology of stress. Current Directions in Psychology Science, 12(4), 124-129.

Ludy-Dobson, C., \& Perry B. D. (2010). The role of healthy relational interaction in buffering the impact of childhood trauma. In E. Gilwith (Ed.), Working with children to heal interpersonal trauma: The power of play (pp. 26-42). New York, NY: Guilford Press. 
Lupien, S. J., de Leon, M., de Santi, S., Convit, A., Tarshi, C., Nair, N. P. V., Thakur, M., McEwen, B. S., Hauger, R. L., \& Meaney, M. J. (1998). Cortisol levels during human aging predict hippocampal atrophy and memory deficits. Nature Neuroscience, 1(4), 6973.

Lyons-Ruth, K. (1996). Attachment relationships among children with aggressive behavior problems: The role of disorganized early attachment patterns. Journal of Consulting and Clinical Psychology, 64(1), 64-73.

Main, M. (1998). De l'attachement à la psychopathologie. Enfance, 51(3), 13-27.

Main, M., Kaplan, N., \& Cassidy, J. (1985). Security in infancy, childhood, and adulthood: A move to the level of representation. Monographs of the Society for Research in Child Development, 50(1-2), 66-104.

Main, M., \& Solomon, J. (1990). Procedures for identifying infants as disorganised/disoriented during the Ainsworth's Strange Situation. In M. T. Greenberg, D. Cicchetti \& E. M. Cummings (Eds.), Attachment in the preschool years: Theory, research, and intervention (pp. 121-160). Chicago, IL: University of Chicago Press.

Minnis, H., Marwick, H, Arthur, J., \& McLaughlin, A. (2006). Reactive Attachment Disorder: A theoretical model beyond attachment. European Child \& Adolescent Psychiatry, 15(6), 336-342.

Newman, L., \& Mares S. (2007). Recent advances in the theories of and interventions with attachment disorders. Child and Adolescent Psychiatry, 20(4), 343-348.

O’Connor, T. G., Bredenkamp, D., \& Rutter, M. (1999). Attachment disturbances and disorders in children exposed to early severe deprivation. Infant Mental Health Journal, 20(1), 1029.

O'Connor, T. G., \& Zeanah C. H. (2003). Current perspectives on attachment disorders: Rejoinder and synthesis. Attachment \& Human Development, 5(3), 321-326.

Oppenheim, D. (2006). Child, parent, and parent-child emotion narratives: Implications for developmental psychopathology. Development and Psychopathology, 18, 771-790.

Perry, B. D. (2001). The neurodevelopmental impact of violence in childhood. In D. Schetky \& E. P. Benedek (Eds.), Textbook of child and adolescent forensic psychiatry (pp. 221-238). Arlington, VA: American Psychiatric Press

Perry, B. D. (2008). Child maltreatment: The role of abuse and neglect in developmental psychopathology. In T. P. Beauchaine \& S. P. Hinshaw (Eds.), Textbook of child and adolescent psychopathology (pp. 93-128). Wiley-Liss, NY: Wiley.

Perry, R., \& Sullivan, R. M. (2014). Neurobiology of attachment to an abusive caregiver: Shortterm benefits and long-term costs. Developmental Psychology, 56(8), 16261634. 
Polan, H. J., \& Hofer, M. R. (2008). Psychobiological origins of infant attachment and its role in development. In J. Cassidy \& P. R. Shaver (Eds.), Handbook of attachment: Theory, research, and clinical applications ( $2^{\text {nd }}$ ed., pp. 158-172). New York, NY: Guilford Press.

Ritchers, M., \& Volkmar F. R. (1994). Reactive Attachment Disorder of infancy or early childhood. Journal of the American Academy of Child and Adolescent Psychiatry, 33(3), 328-332.

Smyke, A. T., Dumitrescu, A., \& Zeanah, C. H. (2002). Attachment disturbances in young children, I: The continuum of caretaking casualty. Journal of the American Academic Child and Adolescent Psychiatry, 41(8), 972-982.

Splaun, A. K. (2012). The brief attachment-focused coding system for story stems: A validity study de doctorat) Repéré dans http://ezproxy.usherbrooke.ca/login?url=https://search-proquestcom.ezproxy.usherbrooke.ca/docview/1022644818? accountid=13835

Steele, M., Steele, H., Woolgar, M., Yabsley, S, Fonagy, P., Johnson, D., \& Croft, C. (2003). An attachment perspective on children's emotion narratives: links across generations. In R. N. Emde, P. F. Wolf \& D. Oppenheim (Eds.), Revealing the inner worlds of young children (pp. 163-181). New York, NY: Oxford University Press.

Thomas, K. M., Drevets, W. C., Dahl, R. E., Ryan, N. D., Birmaher, B., E., Clayton, H., D., Whalen, P. J., \& Casey, B. J. (2001). Amygdala response to fearful faces in anxious and depressed children. Archives of General Psychiatry, 58(11), 1057-1063.

van IJzendoorn, M. H. \& Bakermans-Kranenburg, M. J. (2003). Attachment disorders and disorganised attachment: Similar and different. Attachment \& Human Development, 5(3), 313-320.

van IJzendoorn, M. H., \& Kroonneberg, P. M. (1988). Cross-cultural patterns of attachment: A meta-analysis of the strange situation. Child Development, 59(1), 147-156.

van IJzendoorn, M. H., Schuengel, C., \& Bakermans-Kranenburg, M. J. (1999). Disorganized attachment in early childhood: Meta-analysis of precursors, concomitants, and sequelae. Development and Psychopathology, 11(2), 225-249.

Zeanah, C. H., Boris, N. W., \& Larrieu, J. A. (1997). Infant development and developmental risk: A review of the past 10 Years. Journal of the American Academy of Child \& Adolescent Psychiatry, 36(2), 165-178.

Zeanah, C. H., Boris, N. W., \& Scheeringa, M. S. (1997). Psychopathology in infancy. Journal of Child Psychology \& Psychiatry \& Allied Disciplines, 38(1), 81-99.

Zeanah, C. H., Smyke, A. T., Koga, S. F., \& Carlson, E. (2005). Attachment in institutionalized and community children in Romania. Child Development, 76(5), 1015-1028. 


\section{Appendices}


Appendice A

Exemple d'une histoire des « Histoires à compléter : Histoires sélectionnées par Allison Splaun pour utilisation avec l'Attachement-Focused Coding System »

(AFCS; Reiner \& Keisler Splaun, 2008) 


\section{HISTOIRES À COMPLÉTER}

Histoires sélectionnées par Allison Splaun pour utilisation avec l'Attachement-Focused Coding System (AFCS; Reiner \& Keisler Splaun, 2008)

Traduction en français par Claud Bisaillon, Julie Achim, Natalie Mikic et Miguel M. Terradas (2012)

Université de sherbrooke 
Histoire 1 - Jus renversé

\section{Matériel nécessaire :}

Personnages : Mère, père, enfant 1 et enfant 2

Accessoires : Table, chaises, verre, pichet

Disposition : Les quatre personnages sont assis sur des chaises disposées autour de la table. Chacun d'entre eux a un verre sur la table. Le pichet est également déposé sur la table.

\section{Introduction de l'histoire :}

Dans cette histoire, la famille a soif et ils vont boire du jus. Ils sont tous assis autour de la table en train de boire du jus quand Enfant 1 (à nommer) se lève et se penche au dessus de la table (montrer Enfant 1 en train de le faire) et Oh! Oh! Elle (il) a renversé le jus partout sur le plancher.

Maintenant, montre-moi et raconte-moi ce qui arrive?

\section{Relances :}

1. Si rien n'est dit à l'égard du jus, l'expérimentateur doit demander : Qu'est-ce qui arrive avec le fait que Enfant 1 (à nommer) ait renversé le jus? Qui nettoie?

2.

3. Optionnel : Comment se sentent maman et papa par rapport au fait que Enfant 1 (à nommer) ait renversé le jus? Comment ça? 


\section{Ensemble complet des jouets nécessaires pour les Histoires d'attachement à compléter}

À noter : Les personnages utilisés sont des figurines Playmobil; la taille des accessoires utilisés devrait être à l'échelle de ces figurines.

Personnages : $\quad 1 \mathrm{x}$ mère

$1 \mathrm{x}$ père

$3 \mathrm{x}$ enfant fille

$3 \mathrm{x}$ enfant garçon

Accessoires : $\quad 1$ x sofa

$1 \mathrm{x}$ lit simple

$1 \mathrm{x}$ table de salle à manger

$4 \times$ chaises/tabourets (pour utiliser avec la table)

$1 \mathrm{x}$ cuisinière

$1 \mathrm{x}$ pichet*

$4 \mathrm{x}$ verres/gobelets* 
$1 \mathrm{x}$ poêlon

$1 \mathrm{x}$ tablette/armoire de salle de bain

1 x lavabo de salle de bain

$1 \mathrm{x}$ faux pansement* 
Appendice B

Liste de vérification du comportement des jeunes de 6 à 18 ans (Child Behavior Check-list, CBCL; Achenbach \& Rescorla, 2001) 
Document retiré pour respect du droit d'auteur 
Document retiré pour respect du droit d'auteur 
Document retiré pour respect du droit d'auteur 
Document retiré pour respect du droit d'auteur 